\author{
Maestría en Economía \\ Facultad de Ciencias Económicas \\ Universidad Nacional de La Plata
}

TESIS DE MAESTRIA

\begin{abstract}
ALUMNO
Martín Cicowiez

TITULO

Comercio y Desigualdad Salarial en Argentina: Un enfoque de Equilibrio

General Computado

DIRECTOR

Chisari Omar

FECHA DE DEFENSA

$12 / 7 / 2001$
\end{abstract}


COMERCIO Y DESIGUALDAD SALARIAL EN ARGENTINA: UN ENFOQUE DE EQUILIBRIO GENERAL COMPUTADO

\section{MARTIN CICOWIEZ}

TESIS DE MAESTRIA

MAESTRIA EN ECONOMIA

UNIVERSIDAD NACIONAL DE LA PLATA

DIRECTOR DE TESIS

OMAR O. CHISARI 


\title{
COMERCIO Y DESIGUALDAD SALARIAL EN ARGENTINA: UN ENFOQUE DE EQUILIBRIO GENERAL COMPUTADO
}

\author{
MARTIN CICOWIEZ
}

26-11-2001

\section{RESUMEN}

En este trabajo, se utiliza un modelo de Equilibrio General Computado (CGE) para analizar el efecto de la apertura comercial sobre el salario de los trabajadores calificados versus el salario de los trabajadores no calificados en la Argentina. El resultado que se obtiene es que en el caso de Argentina, el comercio sólo explica una pequeña porción (alrededor de 3\%) del incremento en la desigualdad salarial. Adicionalmente, se presenta una metodología que puede emplearse para realizar ejercicios de descomposición con un modelo de CGE.

\section{INTRODUCCION}

Durante las décadas del ochenta y noventa la desigualdad salarial entre trabajadores calificados y no calificados aumentó considerablemente en varios países ${ }^{1}$. Este aumento ha sido bien documentado para los países de la OECD, en especial Estados Unidos y Gran Bretaña. En el caso de la Argentina, se registra un aumento de la desigualdad salarial durante la década del noventa (Gasparini et al., 2001).

En la literatura económica se mencionan cuatro explicaciones principales para el aumento en la desigualdad salarial: el aumento del comercio internacional de bienes intensivos en mano de obra no calificada (en especial, bienes producidos en países con bajos salarios), el cambio tecnológico sesgado a favor de los trabajadores con mayor calificación, la disminución de la importancia de las instituciones que limitan el mercado (caída del salario mínimo y disminución del poder sindical) y el cambio en la estructura productiva (desindustrialización). De estas cuatro explicaciones, las que más atención han recibido en la literatura son el incremento del comercio y el cambio tecnológico. En cuanto a la importancia relativa de estos dos factores, la mayoría de los autores señalan al cambio tecnológico como causa principal

\footnotetext{
${ }^{1}$ Para un resumen de la literatura sobre este tema, puede consultarse Burtless (1995) y Richardson (1995).
} 
del aumento de la desigualdad salarial ${ }^{2}$. Estos autores señalan que sólo el cambio tecnológico sesgado contra los trabajadores no calificados es consistente con un aumento del diferencial salarial y un aumento simultáneo en la intensidad de uso del trabajo calificado. Esta literatura emplea una gran variedad de métodos econométricos.

En este trabajo se emplea un modelo de Equilibrio General Computado (CGE) para determinar cuánto del cambio en el diferencial salarial entre trabajadores calificados y no calificados observado durante la década del noventa en Argentina puede ser explicado por la apertura comercial ocurrida durante el mismo período. Se elige la década del noventa porque es durante esos años que el aumento del diferencial salarial entre trabajadores con distinta calificación se ve acompañado de un proceso de apertura comercial importante. Adicionalmente, siguiendo a la literatura, se supone que el otro factor que explica el aumento de la desigualdad salarial es el cambio tecnológico y se calcula el cambio tecnológico que, combinado con la apertura comercial, hace que el modelo replique el aumento de la desigualdad salarial ocurrido durante la década del noventa en Argentina.

La aplicación de un modelo de CGE al estudio de este problema se basa en la propuesta de Abrego y Whalley (1999 y 2000). Se emplean dos modelos alternativos para realizar los ejercicios de simulación. El primero es un modelo similar al empleado por Abrego y Whalley y el segundo es un modelo de CGE estático estándar más general. Aunque se trata de modelos muy simples, constituyen una primera aproximación al problema en el marco de un modelo de CGE calibrado con información para Argentina.

El resto del trabajo se organiza de la siguiente manera. La Sección II presenta evidencia empírica para la Argentina sobre la evolución de la desigualdad salarial y de la política comercial durante la década del noventa. La Sección III describe la metodología del CGE. En la Sección IV se describen los modelos empleados. La Sección V presenta la información utilizada para hacer operacional (calibrar) el modelo de CGE. La Sección VI presenta los resultados obtenidos. Finalmente, la Sección VII concluye.

\footnotetext{
${ }^{2}$ Dos excepciones a esta conclusión son Wood (1995 y 1997) y Feenstra y Hanson (1996 y 1999).
} 


\section{EVIDENCIA EMPIRICA PARA ARGENTINA}

\section{II.1. EVOLUCION DEL DIFERENCIAL SALARIAL}

En este trabajo se toma como medida del diferencial salarial entre trabajadores con distinta calificación el cociente entre el salario promedio de los trabajadores calificados y el salario promedio de los trabajadores no calificados ${ }^{3}$. En el caso de la Argentina, la evidencia muestra una caída de este diferencial salarial desde fines de la década del ochenta hasta comienzos de la década del noventa y un aumento a partir de entonces y hasta el año 1998. La fuente de información utilizada para calcular este cociente es la Encuesta Permanente de Hogares $(\mathrm{EPH})$ para el Gran Buenos Aires elaborada por el INDEC ${ }^{4}$. Entre 1992 y 1998 este cociente aumentó alrededor de $10 \%{ }^{5}$. El Gráfico 1 permite apreciar este comportamiento del diferencial salarial.

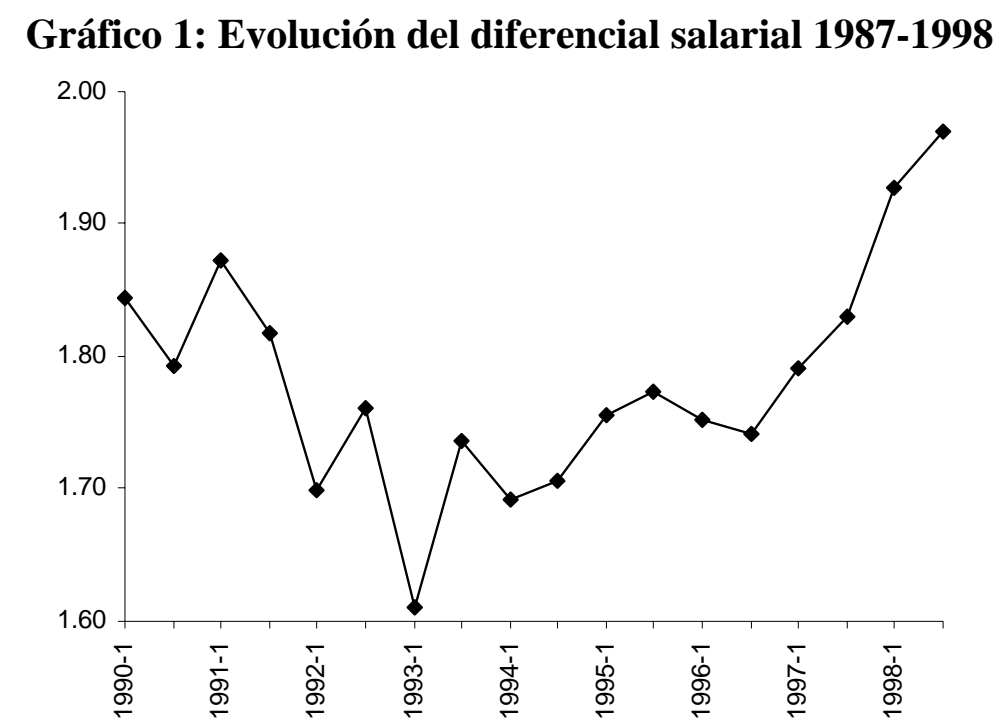

Hacia finales de la década del ochenta la Argentina comienza un proceso de apertura comercial. El arancel promedio pasó de 30,8\% en 1988 a 14,1\% en 1997. Además, en el mismo período, el valor de las exportaciones (importaciones) se multiplicó,

\footnotetext{
${ }^{3}$ Se considera que un trabajador calificado es aquel que tiene educación secundaria completa, universitaria incompleta o universitaria completa. Los resultados no cambian considerablemente si se considera la clasificación de calificados y no calificados que utiliza el INDEC desde 1993. Sin embargo, cuando se utiliza esta clasificación alternativa no se observa la aceleración del aumento en el diferencial salarial a partir de 1997 que si se aprecia en el Gráfico 1.

${ }^{4}$ El Gran Buenos Aires es un aglomerado urbano con una población de 12 millones de habitantes; alrededor de un tercio de la población total del país.

${ }^{5}$ Este comportamiento del diferencial salarial es consistente con el de otras medidas de desigualdad durante el mismo período (Bebczuk y Gasparini, 2001).
} 
aproximadamente, por 2,5 $(4,5)$ indicando un aumento considerable del nivel de integración comercial de Argentina con el resto del mundo.

Dos trabajos que estudian el efecto del aumento del comercio internacional sobre la desigualdad salarial entre trabajadores calificados y no calificados en Argentina son Galiani y Sanguinetti (2000) y Porto (2000). El resultado que obtiene el primero es que el aumento del comercio internacional sólo explica alrededor del 10\% del incremento de la desigualdad salarial. El segundo, en cambio, obtiene que -bajo ciertos supuestos- la apertura comercial podría explicar hasta un 50\% del aumento de la desigualdad salarial. Los trabajos citados emplean metodologías distintas.

\section{II.2. LA POLITICA COMERCIAL ARGENTINA DURANTE LOS NOVENTA}

En términos generales, la Argentina inició un proceso de apertura comercial de manera unilateral hacia finales de la década del ochenta a través de la eliminación de aranceles y cuotas. Sin embargo, esta primera etapa del proceso de apertura no gozó de la credibilidad necesaria para que tuviera efectos sobre la asignación de recursos. Es sólo a partir de 1992 que se genera un proceso de apertura e integración que tuvo consecuencias asignativas importantes (Cristini, 1999).

La apertura comercial de los noventa se llevó a cabo a través de una baja de aranceles y de la eliminación de restricciones cuantitativas. Además de la apertura comercial unilateral, en el año 1991 la Argentina comenzó un proceso de integración regional con la creación del Mercosur. Durante los noventa, los países del Mercosur eliminaron la mayor parte de las barreras al comercio entre ellos y adoptaron un arancel externo común y lograron establecer una unión aduanera "casi perfecta”,

Además de los cambios en la política comercial, en la década del noventa se produjeron otros cambios importantes en la política económica argentina: ordenamiento fiscal, ordenamiento monetario, privatizaciones, apertura financiera, desregulación de la actividad privada. La

\footnotetext{
${ }^{6}$ Para una descripción del proceso de apertura argentino comenzado hacia finales de los ochenta puede consultarse Berrettoni y Cicowiez (2001).
} 
inflación se redujo hasta niveles internacionales y el ingreso per cápita aumento más de 50\% durante la década ${ }^{7}$.

Como ya se mencionó, el cambio tecnológico es el factor que más atención ha recibido en la literatura como causante del aumento de la desigualdad salarial. Aunque es difícil encontrar evidencia directa sobre este factor, existen razones para creer que ha sido importante en la Argentina durante el período considerado. En este sentido, durante la década del noventa se produjo un importante proceso de incorporación de tecnología a través de la privatización de empresas proveedoras de servicios públicos y de la importación de bienes de capital y de insumos intermedios.

\section{METODOLOGIA}

La metodología utilizada en este trabajo es la del Equilibrio General Computado (CGE). En esta sección se presentan las principales características de los modelos de CGE. Un modelo de equilibrio general tiene en cuenta que un cambio exógeno (de política o de otro tipo) que afecta directamente a una parte de la economía puede tener repercusiones a través de toda la economía. En consecuencia, debido a que capta el impacto de la reasignación de recursos entre los distintos sectores de una economía, este tipo de modelos es una herramienta ideal para identificar ganadores y perdedores luego de un cambio de política.

Un modelo de CGE walrasiano hace operacional la estructura de equilibrio general de Walras. Las características de un modelo de equilibrio general walrasiano son las que se mencionan a continuación. El número de consumidores existentes en el modelo debe estar especificado. Cada uno de ellos tiene una dotación inicial de bienes y un conjunto de preferencias que resultan en funciones de demanda para cada uno de los bienes. Las demandas de mercado son las sumas de las demandas individuales. Estas demandas de mercado dependen de todos los precios, son continuas, no negativas, homogéneas de grado cero (no hay ilusión monetaria) y satisfacen la ley de Walras (a cualquier conjunto de precios el valor del gasto de los consumidores se iguala con su ingreso). Del lado de la producción, la tecnología se describe mediante funciones de producción con rendimientos constantes (o no crecientes) a escala. Los productores maximizan sus beneficios. La homogeneidad de grado cero de las funciones de

\footnotetext{
${ }^{7}$ Para un resumen de los cambios ocurridos en la economía argentina en este período puede consultarse Bebczuk y Gasparini (2001).
} 
demanda y la homogeneidad de grado uno en precios de los beneficios (si los precios se duplican se duplican los beneficios monetarios) implican que sólo los precios relativos son relevantes en este modelo; el nivel absoluto de precios no tiene ningún impacto sobre los resultados del modelo. El equilibrio de un modelo como este se caracteriza por un conjunto de precios y niveles de producción que igualan la oferta y la demanda para todos los bienes (Shoven y Whalley, 1984).

Entonces, los modelos de CGE son la contraparte numérica de los modelos de equilibrio general walrasianos tipo Arrow-Debreu (1954) y están basados en el comportamiento optimizador de los agentes económicos (los consumidores maximizan su utilidad y los productores maximizan sus beneficios). Las aplicaciones numéricas del equilibrio general se iniciaron con el trabajo de Harberger (1962) sobre incidencia tributaria en el contexto de un modelo numérico de dos sectores. El trabajo de Scarf (1969) hizo posible la determinación del equilibrio de un sistema walrasiano. La utilización de modelos de CGE recibió un impulso fundamental del trabajo pionero de Shoven y Whalley (1972, 1984, 1992). Más recientemente, contribuciones como las del GTAP (Hertel, 1997) y Rutherford (1999) han contribuido al desarrollo y utilización de esta metodología. El objetivo de los trabajos que emplean modelos de CGE es analizar los efectos cuantitativos de cambios exógenos sobre la asignación óptima de recursos, la eficiencia y el bienestar. Dos de los campos de mayor aplicación han sido las finanzas públicas (por ejemplo, la evaluación de sistemas tributarios alternativos) y el comercio internacional (en especial, la evaluación de distintos acuerdos comerciales). Actualmente, los modelos de CGE son ampliamente utilizados para el análisis cuantitativo de políticas económicas.

La idea básica detrás de un modelo de CGE es simple. Se construye un modelo matemático de una economía y se recopila información para un período de tiempo dado. Luego, las características de la economía en ese período de tiempo son utilizadas para resolver el modelo numéricamente. Este último paso se realiza utilizando una computadora. Un modelo de CGE tiene una estructura transparente y consistente con la teoría económica y es una herramienta ideal para evaluar políticas alternativas. En un análisis de equilibrio general se modela toda la economía. En cambio, en un análisis de equilibrio parcial se analiza la situación de un mercado en particular suponiendo constantes las condiciones en el resto de los mercados de la economía. 
Resumiendo, un modelo de CGE como el que se emplea en este trabajo es una representación en computadora de una economía y posee las siguientes características:

i. Hay varios agentes económicos que interactúan.

ii. El comportamiento individual está basado en la optimización microeconómica. (Dos problemas típicos para ilustrar este punto son la maximización de la utilidad de los consumidores y la maximización de beneficios de las firmas).

iii. La mayoría de las interacciones entre los agentes se realizan a través de mercados y precios.

iv. Son modelos típicamente desagregados, con varios agentes y mercados.

v. Los datos empleados en su construcción son pocos cuando se los compara con el número de parámetros de comportamiento y tecnológicos del modelo. Normalmente, la información para un modelo de CGE corresponde a un "equilibrio general" observado o caso base y a un conjunto de estimaciones independientes de elasticidades de oferta y demanda. Típicamente, un modelo de CGE se construye de manera tal que replique las transacciones observadas en el caso base.

vi. Típicamente, las asignaciones de equilibrio no pueden caracterizarse fácilmente como la solución a un único problema de optimización (el de un planificador central).

vii. La formulación de este tipo de modelos tiene como objetivo (implícito o explícito) el análisis de políticas económicas.

Los pasos “normales” a seguir en un estudio de CGE son:

i. Diseño general. Con base en el problema de política a tratar y en la disponibilidad de datos, se determinan las dimensiones básicas del análisis: número de regiones, de consumidores, de sectores productivos, de factores, etc.

ii. Diseño de los problemas de elección individuales. Especificar para cada agente del modelo (familias, empresas y gobierno) el problema de elección que deberá enfrentar. Estos problemas podrán ser simples (por ejemplo, un gobierno que sólo transfiere lo recaudado por impuestos a los consumidores) o complicados (por ejemplo, un 
consumidor que debe asignar su ingreso entre distintos bienes de manera tal de maximizar su utilidad respetando su restricción presupuestaria).

iii. Funciones de oferta y demanda. Resolver los problemas de elección individuales del modelo. Típicamente, se trata de solucionar problemas de optimización restringida (maximización de la utilidad de los consumidores y minimización del costo de las firmas).

iv. Desarrollar un sistema de notación. Se debe tener especial cuidado en las correspondencias entre mercados y precios. Selección de sets y nombres para los precios de los bienes y factores. Suele ser conveniente el empleo de más de un precio para un mismo bien si ese bien está sujeto al pago de impuestos.

v. Calibración. Se trata de inferir el valor de los parámetros de las ecuaciones de comportamiento de manera tal que el caso base (el equilibrio observado) sea una solución del modelo. En este procedimiento, se combinan las ecuaciones de comportamiento con las elecciones de los agentes en el caso base y con valores para las elasticidades (parámetros libres).

vi. Programación del modelo. Codificar el sistema de ecuaciones no lineales que representa el modelo. En este estudio, para la solución del sistema de ecuaciones no lineales que representa el modelo se utiliza el software GAMS (General Algebraic Modeling System) documentado en Brooke et al. (1996).

vii. Replicar el caso base. Debido a que los parámetros del modelo fueron obtenidos mediante un proceso de calibración, si se asignan los valores del caso base a los parámetros y variables exógenas, el modelo debería dar el caso base como solución. Este paso es útil para confirmar la correcta codificación del modelo.

viii. Realizar simulaciones. Finalmente, con el modelo correctamente codificado y calibrado se modifica el valor de alguna variable exógena o parámetro, se recalcula el equilibrio y se analizan los resultados a partir de la comparación con el caso base.

La metodología que se emplea en los modelos de CGE para analizar los efectos económicos de políticas comerciales alternativas es la realización de experimentos contrafactuales o simulaciones. Se pregunta al modelo que hubiese pasado en el año base si hubiese sido 
implementada la política comercial de interés y el resto de las políticas domésticas (fiscal y monetaria) y las condiciones externas (el comportamiento de los precios mundiales) permanecieran igual. Por lo tanto, este tipo de análisis enfatiza los efectos de la política comercial aislándola de otros factores. Debido a que no se incorporan en el análisis los cambios esperados en esos otros factores, no se trata de una predicción. Finalmente, un modelo de CGE estático no incorpora características dinámicas. Las características mencionadas deben ser tenidas en cuenta cuando se interpretan los resultados de un modelo de CGE.

\section{MODELOS}

En esta sección se presentan los modelos utilizados en los ejercicios de simulación. En primer lugar se presenta el modelo más simple y luego el modelo más general.

\section{IV.1. MODELO SIMPLE}

La teoría más frecuentemente citada para explicar el nexo entre el comercio y los salarios es el modelo de Heckscher-Ohlin (HO) de comercio internacional ${ }^{8}$. Este modelo explica los patrones de comercio internacional a partir de las diferencias relativas en la dotación de factores de producción de los socios comerciales. El modelo predice que entre dos países, A y $\mathrm{B}$, que comparten la misma tecnología, el país A exportará bienes que sean producidos con relativamente más del factor de producción que es relativamente abundante en el país A e importará bienes producidos con relativamente más del factor de producción que es relativamente abundante en el país B. Del modelo de HO se derivan dos teoremas acerca del efecto del comercio sobre el precio de los factores productivos: (i) El teorema de igualación del precio de los factores afirma que bajo los supuestos del modelo de $\mathrm{HO}$ y un régimen de libre comercio, el precio de los factores de producción será el mismo en ambos socios comerciales y (ii) El teorema de Stolper-Samuelson afirma que un incremento en el precio doméstico de un bien debido a un incremento del arancel o a una mayor protección aumentará

\footnotetext{
${ }^{8}$ Los supuestos de la versión más sencilla de este modelo son los siguientes: (i) hay dos países, (ii) cada país produce dos bienes, (iii) la producción de cada bien requiere de dos factores de producción, (iv) cada país tiene una dotación fija de cada uno de los factores, (v) los factores son perfectamente móviles entre sectores y perfectamente inmóviles entre países, (vi) los dos países emplean la misma tecnología de producción, (vii) para todos los precios relevantes de los factores, uno de los bienes es siempre relativamente intensivo en el uso de uno de los factores, (viii) uno de los países es relativamente abundante en uno de los factores y (ix) los costos de transporte son nulos.
} 
el precio real del factor de producción utilizado relativamente con mayor intensidad en la producción de ese bien.

En nuestro caso, si Argentina es un país relativamente abundante en mano de obra calificada (Cristini, 1999), se esperaría un incremento del salario relativo de este tipo de trabajo luego de la apertura comercial. Sin embargo, es importante tener en cuenta que Argentina es relativamente más abundante en tierra y que es un exportador neto de bienes intensivos en recursos naturales y un importador neto de bienes intensivos en trabajo calificado y tecnología (Berlinski, 1998).

Sin embargo, como demuestran Abrego y Whalley (1999), el modelo de HO sólo puede ser resuelto (numéricamente) en presencia de shocks (por ejemplo, caída de las tasas arancelarias) relativamente pequeños. Cuando se emplean las formas funcionales usuales y las dotaciones factoriales están fijas, la frontera de producción es casi lineal (Johnson, 1966). Por lo tanto, incluso shocks pequeños son acompañados de especialización en la producción ${ }^{9}$.

En consecuencia, el primer modelo que se utiliza para determinar el efecto de la apertura comercial sobre la desigualdad salarial es una extensión del modelo de HO en el que se suprime el supuesto de bienes homogéneos. El modelo HO es un caso particular del que se utiliza a continuación ${ }^{10}$.

\section{ESTRUCTURA DEL MODELO SIMPLE}

El modelo es de una economía abierta y pequeña, que toma como dados los precios de sus exportaciones y de sus importaciones ${ }^{11}$. Sin embargo, se supone que los precios mundiales no dominan el sistema de precios doméstico. Existen tres bienes, dos factores productivos y un consumidor representativo dueño de toda la dotación factorial del país que se supone fija. Dos de los bienes se producen en el país doméstico y el tercero se importa desde el resto del mundo. El país doméstico produce un bien exportable y un bien doméstico no transable que es

\footnotetext{
9 Se podría utilizar una variante del modelo con factores específicos para eliminar el problema de la especialización. Sin embargo, en un modelo de este tipo los shocks de precios son soportados, en su mayor parte, por los factores específicos más que por los factores móviles (trabajo calificado y no calificado).

${ }^{10}$ Como se hará evidente más adelante, esto ocurre cuando la elasticidad de sustitución (Armington) entre el bien doméstico y el importado es igual a infinito.

${ }^{11}$ En el modelo de HO de dos países, dos bienes y dos factores, la abundancia relativa de factores determina el patrón de comercio. El modelo que se utiliza aquí contiene sólo un país pequeño que toma como dados los
} 
sustituto imperfecto del bien importado. A continuación se detalla la estructura de este modelo. Para el lector interesado, en las notas al pie de página, se presenta la formulación algebraica de las formas funcionales empleadas.

\section{Lado del consumo}

La utilidad del consumidor representativo depende del consumo de tres bienes: el bien exportable $E$, el bien doméstico no transable, $D$, y el bien importado, $M$. Se supone que el consumidor representativo maximiza su utilidad en dos etapas. En la primera elige entre consumir el bien exportable y un bien compuesto, $A$, formado por el bien doméstico y el bien importado. En la segunda etapa determina la composición óptima del bien compuesto. Es decir, determina cuanto de bien doméstico y cuanto de bien importado consume. Las preferencias se representan con una función de utilidad tipo Cobb-Douglas (CD) ${ }^{12}$ para la primera etapa y tipo $\mathrm{CES}^{13}$ (Elasticidad de Sustitución Constante) para la segunda. Cuando el consumidor distingue entre bienes nacionales y bienes importados (por ejemplo, un auto nacional es distinto de un auto japonés) se dice que tiene preferencias tipo Armington (1969). Matemáticamente, en el nivel superior de la función de utilidad el consumidor representativo resuelve el siguiente problema:

$$
\begin{aligned}
& \max U=C D\left(A^{d}, E^{d}\right) \\
& \text { s. a. } I=\overline{P_{E}} E^{d}+P_{A} A^{d}
\end{aligned}
$$

donde $U$ es una función de utilidad tipo Cobb-Douglas, $A^{d}$ es la cantidad demandada de bien compuesto $A, E^{d}$ es la cantidad demandada de bien $E, \overline{P_{E}}$ es el precio de $E, P_{A}$ es el precio del bien compuesto A y I es el ingreso del consumidor representativo y está dado por

precios de sus exportaciones e importaciones. El patrón de comercio del equilibrio inicial está determinado por la ventaja comparativa propia del país doméstico, no la abundancia relativa de factores.

${ }^{12}$ Algebraicamente, la función de utilidad tipo Cobb-Douglas puede escribirse como

$$
C D\left(A^{d}, E^{d}\right)=\left(A^{d}\right)^{\alpha_{A}}\left(E^{d}\right)^{\alpha_{E}}
$$

donde $\alpha_{A} y \alpha_{E}$ son parámetros de distribución.

${ }^{13}$ Algebraicamente, la función tipo CES puede escribirse como

$$
\operatorname{CES}\left(M^{d}, D^{d}\right)=\gamma\left[\beta_{M}\left(M^{d}\right)^{\frac{\varepsilon-1}{\varepsilon}}+\beta_{D}\left(D^{d}\right)^{\frac{\varepsilon-1}{\varepsilon}}\right]^{\frac{\varepsilon}{\varepsilon-1}}
$$

donde $\gamma$ es un parámetro de escala, $\beta$ es un parámetro de distribución y $\varepsilon$ es la elasticidad de sustitución entre el bien importado y el bien doméstico. 


$$
I=w_{L} \bar{L}+w_{H} \bar{H}+R+\bar{B}
$$

donde $\bar{L}$ es la dotación (exógena) de trabajo no calificado, $\bar{H}$ es la dotación (exógena) de trabajo calificado, $R$ es la transferencia que recibe del gobierno, $\bar{B}$ es la transferencia que recibe desde el resto del mundo que se supone fija e igual al déficit comercial, $w_{L}$ es el salario del trabajo no calificado y $w_{H}$ es el salario del trabajo calificado. Resolviendo las Condiciones de Primer Orden (CPO) del problema anterior, se obtienen las demandas de bien $A, A^{d}$, y de bien $E, E^{d}$.

Como se mencionó más arriba, el bien compuesto es un bien agregado tipo Armington. El consumidor debe determinar cuál es la composición óptima (cuánto de bien importado y cuánto de bien doméstico consume) que le permite minimizar el gasto total en el bien compuesto. Analíticamente, el consumidor representativo resuelve el siguiente problema:

$$
\begin{aligned}
& \min P_{A} A^{d}=\overline{P_{M}}(1+\tau) M^{d}+P_{D} D^{d} \\
& \text { s. a. } A^{d}=C E S\left(M^{d}, D^{d}\right)
\end{aligned}
$$

donde el bien compuesto $A$ se modela con una función tipo CES de la cantidad demandada de bien $M, M^{d}$, y de la cantidad demandada de bien $D, D^{d}, \overline{P_{M}}$ es el precio del bien $M, P_{D}$ es el precio del bien $D$ y $\tau$ es la tasa del arancel a las importaciones. El precio $P_{A}$ se obtiene de la condición de igualdad entre el gasto total en $A$ y la suma del gasto en $M$ y en $D$. De las CPO del problema anterior surgen las funciones de demanda $M^{d}$ y $D^{d}$.

\section{Lado de la producción}

En este primer modelo se producen sólo dos bienes: el exportable $E$ y el doméstico no transable $D$. En la producción sólo se utiliza trabajo no calificado $L$ y trabajo calificado $H$. La tecnología es de Rendimientos Constantes a Escala y se modela con funciones de producción tipo $\mathrm{CES}^{14}$ en los dos sectores. El problema de minimización de costos que resuelve el productor de bien $D$ es

\footnotetext{
${ }^{14}$ Algebraicamente, la función de producción tipo CES puede escribirse como
} 
$\min w_{L} L_{D}+w_{H} H_{D}$

s. a. $D^{s}=C E S\left(L_{D}, H_{D}, \pi\right)$

donde $D^{S}$ es la oferta de bien $D, L_{D}$ es el empleo de $L$ en la producción de bien $D, H_{D}$ es el empleo de $H$ en la producción de bien $D$ y $\pi$ es el cambio tecnológico. Resolviendo las CPO, se obtienen las demandas de factores para la producción de $D, L_{D}$ y $H_{D}$.

La producción del bien $E$ se modela de manera similar a la producción del bien $D$. El productor resuelve el siguiente problema de minimización de costos:

$\min w_{L} L_{E}+w_{H} H_{E}$

s. a. $E^{s}=C E S\left(L_{E}, H_{E}, \pi\right)$

donde la notación utilizada es similar al caso anterior. Al igual que en el caso anterior, de la resolución de este problema se obtienen las demandas de factores en la producción de bien $E$, $L_{E}$ y $H_{E}$.

\section{Gobierno}

En este modelo, el gobierno sólo recauda lo producido por el arancel y lo devuelve al consumidor como una transferencia de suma fija. La restricción presupuestaria del gobierno es, entonces,

$$
\tau \overline{P_{M}} M^{d}=R
$$

donde $R$ es la recaudación total del arancel.

$$
C E S(L, H)=\phi\left[\pi \delta L^{\frac{\Omega-1}{\Omega}}+(1-\pi \delta) H^{\frac{\Omega-1}{\Omega}}\right]^{\frac{\Omega}{\Omega-1}}
$$

donde $\phi$ es un parámetro de escala, $\delta$ es un parámetro de distribución, $\Omega$ es la elasticidad de sustitución entre el trabajo no calificado y el trabajo calificado y $\pi$ es el cambio tecnológico. 


\section{Condiciones de equilibrio}

El equilibrio de este modelo se caracteriza por las siguientes condiciones: equilibrio en los mercados de factores, $L_{D}+L_{E}=\bar{L}$ y $H_{D}+H_{E}=\bar{H}$, equilibrio en el mercado del bien $D$, $D^{d}=D^{s}$, equilibrio del sector externo, $\overline{P_{M}} M^{d}=\overline{P_{E}}\left(E^{s}-E^{d}\right)+\bar{B}$ y cero beneficios en la producción, $\overline{P_{E}} E^{s}-w_{L} L_{E}-w_{H} H_{E}=0$ y $P_{D} D^{s}-w_{L} L_{D}-w_{H} H_{D}=0$. Los precios $\overline{P_{M}}$ у $\overline{P_{E}}$ son exógenos y el precio $P_{D}$ es endógeno (a diferencia de un modelo donde $D$ y $M$ son bienes homogéneos o sustitutos perfectos en el consumo).

\section{IV.2. MODELO GENERAL}

En esta sección se presenta un modelo más general que se emplea para analizar los efectos del comercio sobre la desigualdad salarial en Argentina. Se trata de un modelo estático del tipo De Melo y Robinson (1989). La estructura de este modelo es estándar en la literatura que utiliza modelos de CGE. A diferencia del modelo anterior, el Modelo General incorpora diez sectores productivos ${ }^{15} \mathrm{y}$ tres factores primarios. La existencia de diez bienes hace posible incorporar las diferencias en el cambio que experimentaron las distintas tasas arancelarias en el período que se analiza.

La Tabla 1 muestra los sectores considerados en la implementación de este modelo ${ }^{16}$. Además, en la tabla se muestra el índice de Ventajas Comparativas Reveladas (VCR) para cada uno de los sectores. El índice de VCR se define como el cociente entre la participación de un producto en las exportaciones de un país y la participación de ese producto en las exportaciones mundiales. Un valor mayor (menor) a uno indica la presencia (ausencia) de VCR en ese sector.

\footnotetext{
${ }^{15}$ Cada uno de estos sectores productivos produce un único bien.

${ }^{16}$ La composición de cada uno de los diez sectores productivos considerados es la siguiente: Cereales y Ganadería (G_L): Arroz con cáscara, Trigo, Otros cereales, Lana y Otros productos de origen animal. Otros Productos Agrícolas (OAF): Otros cultivos, Silvicultura y Pesca. Industrias Alimenticias (FIN): Arroz procesado, Productos de la carne, Productos de la leche, Otros productos alimenticios y Bebidas y tabaco. Textiles e Indumentaria (TEX): Textiles y Indumentaria. Manufacturas de Recursos Naturales (NRM): Productos de cuero, Productos de madera y Productos del papel y de la industria gráfica. Minería, Petróleo y Carbón (MPC): Carbón, Petróleo, Gas, Otros minerales y Productos del petróleo y del carbón. Transporte (TRN): Equipo de transporte. Industria Química y Otras Industrias (C_O): Químicos, plásticos y goma, Minerales no metálicos y Otras manufacturas. Maquinaria y Productos Metálicos (M_M): Metales ferrosos, Metales no ferrosos, Productos metálicos y Maquinaria y equipo. Servicios (SVC): Electricidad, gas y agua, Construcción, Comercio y transporte, Otros servicios (privados), Otros servicios (gobierno) y Vivienda.
} 
Tabla 1: Sectores considerados y VCR

\begin{tabular}{cclc}
\hline & & \multicolumn{1}{c}{ Descripción } & VCR \\
\hline 1 & G_L & Cereales y Ganadería & 11.3 \\
2 & OAF & Otros Productos Agrícolas & 6.7 \\
3 & FIN & Industrias Alimenticias & 11.7 \\
4 & TEX & Textiles e Indumentaria & 0.4 \\
5 & NRM & Manufacturas de Recursos Naturales & 1.2 \\
6 & MPC & Minería, Petróleo y Carbón & 1.8 \\
7 & TRN & Transporte & 0.4 \\
8 & C_0 & Industria Química y otras Industrias & 0.8 \\
9 & M_M & Maquinaria y Productos Metálicos & 0.4 \\
10 & SVC & Servicios & - \\
\hline
\end{tabular}

Fuente: Elaboración propia en base al GTAP y COMTRADE.

Según el indicador de VCR, Argentina presenta una marcada ventaja comparativa en la producción de productos primarios y alimentos. En términos generales, estos sectores son relativamente intensivos en mano de obra no calificada.

A modo de resumen, el Gráfico 2 describe la estructura del Modelo General utilizado en los ejercicios de simulación.

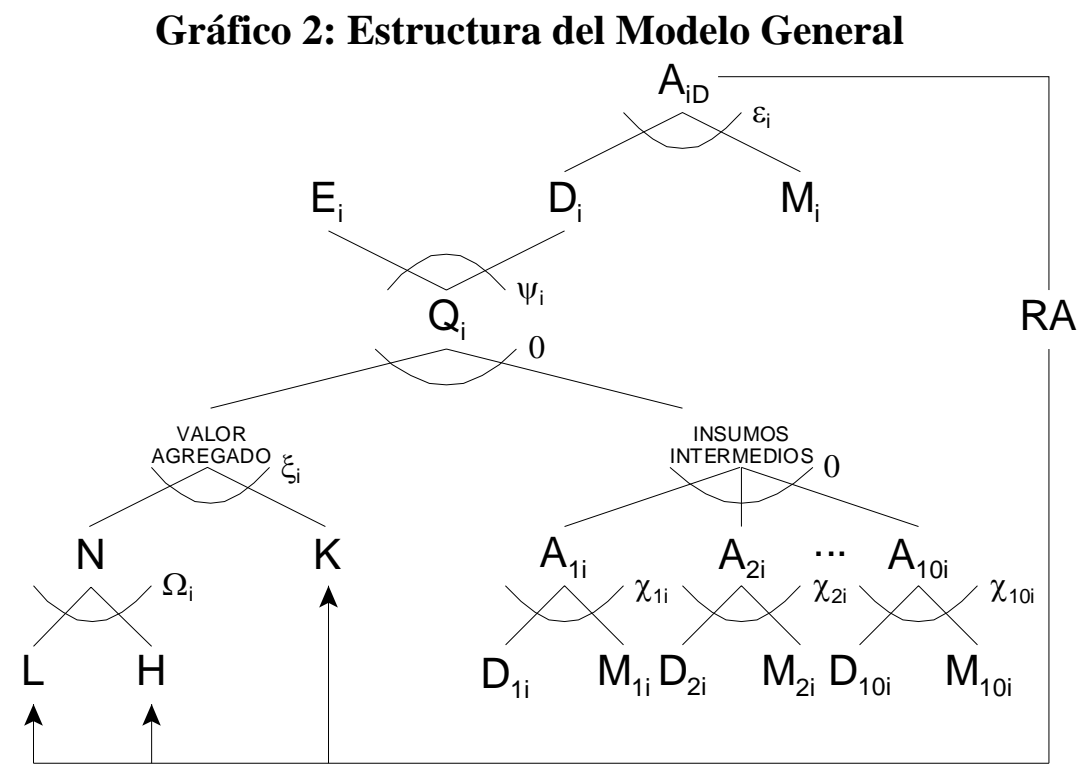

El producto del sector i $\left(\mathrm{Q}_{\mathrm{i}}\right)$ se produce utilizando capital $(\mathrm{K})$, trabajo no calificado $(\mathrm{L})$, trabajo calificado $(\mathrm{H})$ e insumos intermedios representados por agregados Armington $\left(\mathrm{A}_{\mathrm{ji}}\right)$. Utilizando el supuesto de Armington (1969), la demanda intermedia del sector i de bien j se representa con un bien compuesto formado por bienes domésticos $\left(\mathrm{D}_{\mathrm{ji}}\right)$ e importaciones $\left(\mathrm{M}_{\mathrm{ji}}\right)$. Un Agregado Armington también se emplea en el consumo privado $\left(\mathrm{A}_{\mathrm{iD}}\right)$. La producción $\mathrm{Q}_{\mathrm{i}}$ se destina al consumo doméstico de bienes $\left(\mathrm{D}_{\mathrm{i}}\right)$ y a la exportación $\left(\mathrm{E}_{\mathrm{i}}\right)$. La elección de los 
productores entre vender al mercado doméstico o exportar al resto del mundo se modela mediante una función de transformación tipo CET (Elasticidad de Transformación Constante). Se supone la existencia de un agente representativo (RA) que tiene una dotación fija de L, H y $K$, recauda impuestos y demanda $A_{D}$. Se supone que existe pleno empleo de los factores primarios de producción. Los factores primarios de producción son perfectamente móviles entre sectores y tienen una oferta inelástica.

En el gráfico, las letras griegas representan elasticidades y la interpretación de cada una es la siguiente: $\varepsilon_{\mathrm{i}}$ y $\chi_{\mathrm{ji}}$ son las elasticidades de sustitución Armington en el consumo final e intermedio, respectivamente; $\Omega_{\mathrm{i}}$ es la elasticidad de sustitución entre L y H en la producción de i; $\xi_{\mathrm{i}}$ es la elasticidad de sustitución entre $\mathrm{N}$ y K en la producción de i y $\psi_{\mathrm{i}}$ es la elasticidad de transformación entre las ventas al mercado doméstico (D) y las exportaciones (E) de i. Finalmente, la elasticidad de sustitución entre el valor agregado y los insumos intermedios es cero; es decir, el valor agregado y los insumos intermedios se demandan en proporciones fijas del producto.

\section{ESTRUCTURA DEL MODELO GENERAL}

En esta sección se presentan, de manera resumida, las funciones que determinan el comportamiento optimizador de los agentes del Modelo General. Para construir un modelo de CGE es necesario especificar las formas funcionales que caracterizan las preferencias y la tecnología de producción. Al igual que cuando se presentó el Modelo Simple, para el lector interesado, en las notas al pie de página se muestra la formulación algebraica de las formas funcionales. Adicionalmente, el Apéndice A presenta todas las ecuaciones de este modelo de manera análoga a como fueron codificadas en GAMS. La estructura del modelo y las formas funcionales utilizadas en este trabajo son estándar en la literatura que emplea modelos de CGE.

\section{Lado de la producción}

Cada sector productivo i produce dos tipos de bien: uno para el mercado doméstico $\left(D_{i}\right)$ y otro para exportar al resto del mundo $\left(E_{i}\right)$. Se supone que estos bienes son sustitutos imperfectos. Para modelar esta posibilidad se utiliza una función con Elasticidad de Transformación 
Constante $(\mathrm{CET})^{17}$. Para producir, cada sector utiliza trabajo $\left(N_{i}\right)$, capital $\left(K_{i}\right)$ y bienes intermedios $\left(A_{j i}\right)$. El trabajo y el capital son perfectamente móviles entre sectores. La función de producción del sector i es, entonces,

$$
Q_{i}=g\left(D_{i}, E_{i}\right)=f\left\lfloor K_{i}, N_{i}\left(L_{i}, H_{i}\right), A_{j i}\right\rfloor
$$

donde $g$ es la función de transformación y $f$ es la función de producción que convierte factores primarios e insumos intermedios en producto. La función de transformación es

$$
g\left(D_{i}, E_{i}\right)=\operatorname{CET}\left(D_{i}, E_{i}\right)
$$

La función de producción combina valor agregado e insumos intermedios mediante una función tipo Leontief (LF) o de coeficientes fijos. El trabajo y el capital se combinan mediante una función con Elasticidad de Sustitución Constante (CES) ${ }^{18}$ para producir el valor agregado. A su vez, el trabajo total utilizado por cada sector es un agregado tipo CES de trabajo no calificado y trabajo calificado. Por su parte, los insumos intermedios de los distintos sectores son incorporados como un agregado tipo Leontief. La función de producción es, entonces,

$$
f=\left\lfloor K_{i}, N_{i}\left(L_{i}, H_{i}\right), A_{j i}\right\rfloor=\operatorname{LF}\left[\operatorname{CES}\left(K_{i}, N_{i}\right), \operatorname{LF}\left(A_{1 i}, A_{2 i}, \ldots, A_{10 i}\right)\right]
$$

donde $N_{i}$ es, a su vez, igual a

$$
N_{i}=\operatorname{CES}\left(L_{i}, H_{i}\right)
$$

El insumo intermedio producido por el sector $\mathrm{j}$ utilizado en el sector i $\left(A_{j i}\right)$ es un bien compuesto (Armington) por las variedades doméstica $\left(D I_{j i}\right)$ e importada $\left(M I_{j i}\right)$ del bien j. Los

${ }^{17}$ Algebraicamente, la función de transformación tipo CET puede escribirse como

$$
\operatorname{CET}\left(D_{i}, E_{i}\right)=\theta_{i}\left(\eta_{i}^{D} D_{i}^{\frac{1+\psi_{i}}{\psi_{i}}}+\eta_{i}^{E} E_{i}^{\frac{1+\psi_{i}}{\psi_{i}}}\right)^{\frac{\psi_{i}}{1+\psi_{i}}}
$$

donde $\theta_{i}$ es un parámetro de escala, $\eta_{i}$ es un parámetro de distribución y $\psi_{i}$ es la elasticidad de transformación. ${ }^{18}$ Algebraicamente, la función tipo CES puede escribirse como

$$
\operatorname{CES}\left(K_{i}, N_{i}\right)=\mu_{i}\left(v_{i}^{K} K_{i}^{\frac{\xi_{i}-1}{\xi_{i}}}+v_{j}^{N} N_{i}^{\frac{\xi_{i}-1}{\xi_{i}}}\right)^{\frac{\xi_{i}}{\xi_{i}-1}}
$$

donde $\mu_{i}$ es un parámetro de escala, $v_{i}$ es un parámetro de distribución y $\xi_{i}$ es la elasticidad de sustitución entre el capital y el trabajo en la producción del bien i. 
productores consideran a estas variedades como sustitutos imperfectos. Esto se modela mediante una función tipo CES:

$$
A_{j i}=C E S\left(D I_{j i}, M I_{j i}\right)
$$

\section{Lado del consumo}

El agente representativo es dueño de toda la dotación factorial de la economía $(K, L$ y $H)$, recibe -como una transferencia de suma fija- todo lo recaudado por el arancel y demanda bienes. La función de utilidad de este consumidor es de tipo Cobb-Douglas (CD) ${ }^{19}$ y demanda bienes domésticos e importados combinados en un agregado Armington:

$$
U=C D\left(A_{i D}, A_{2 D}, \ldots, A_{10 D}\right)
$$

donde $U$ es la utilidad que el agente representativo deriva de consumir los bienes compuestos tipo Armington $A_{i D}$. Estos bienes compuestos se modelan, al igual que para el consumo intermedio, utilizando una función tipo CES:

$$
A_{i D}=C E S\left(D D_{i D}, M D_{i D}\right)
$$

donde $D D_{i D}\left(M D_{i D}\right)$ es la cantidad de la variedad doméstica (importada) de bien i consumida por el consumidor representativo.

\section{Gobierno}

Al igual que en el Modelo Simple, el gobierno se modela como recaudando lo producido por el arancel y devolviendo el total recaudado al consumidor representativo como una transferencias de suma fija. En consecuencia, el agente representativo consolida las demandas privada, pública y de inversión.

\footnotetext{
${ }^{19}$ Algebraicamente, la función de utilidad tipo Cobb-Douglas puede escribirse como$$
C D\left(A_{1 D}, A_{2 D}, \ldots, A_{10 D}\right)=\prod_{i=1}^{10} A_{i D}^{\alpha_{i}}
$$

donde $\alpha_{i}$ es un parámetro de distribución.
} 


\section{Condiciones de equilibrio}

El equilibrio de este modelo se caracteriza por las siguientes condiciones: Los precios de los factores y los bienes producidos domésticamente son determinados endógenamente. En todos los mercados la oferta se iguala con la demanda. Los beneficios de los productores son cero. Esto significa que los costos de producción de cada sector se igualan con el valor del producto. El consumidor representativo cumple con su restricción presupuestaria. Es decir, el valor de sus gastos se iguala con su ingreso. Este ingreso está dado por la remuneración a los factores productivos que posee más el monto recaudado por los aranceles más la transferencia (igual al déficit comercial) que recibe desde el resto del mundo. Esta transferencia es igual al déficit comercial que se supone fijo en el valor del equilibrio inicial. Asimismo, el sector externo de la economía también se encuentra equilibrado.

\section{MATRIZ DE CONTABILIDAD SOCIAL Y CALIBRACION}

Para la calibración de los modelos se construyó una Matriz de Contabilidad Social (SAM) con información para la Argentina correspondiente al año 1992 y estimaciones econométricas para algunas de las elasticidades del modelo obtenidas de la literatura.

La SAM construida representa el equilibrio inicial o caso base. La información utilizada para construir la SAM se obtuvo (principalmente) de la versión 3 de la base de datos del Global Trade Analysis Project (GTAP). Esta información se complementó con información de la Matriz Insumo-Producto (MIP) argentina de 1997, del Sistema de Cuentas Nacionales (SNC), de la EPH y del TRade Analysis and INformation System (TRAINS). Se utilizan dos versiones de la SAM: una para calibrar el Modelo Simple y otra para calibrar el Modelo General. En el Apéndice B se presenta una descripción detallada de las fuentes de información utilizadas para construir la SAM. Además, se presentan los valores de las elasticidades del modelo.

Una SAM refleja todas las transacciones que tienen lugar en una economía determinada durante un período de tiempo dado (típicamente, un año). En términos generales, una SAM capta el proceso circular de la demanda, que lleva a la oferta, que lleva al ingreso, que lleva nuevamente a la demanda.

Para construir una SAM que permita calibrar un modelo de CGE es necesario suponer que los valores observados de las variables constituyen un “equilibrio general”. Es decir, deben 
cumplirse las siguientes condiciones: (i) las demandas se igualan a las ofertas en todos los mercados, (ii) ningún sector productivo tiene beneficios positivos, (iii) todos los agentes modelados cumplen con su restricción presupuestaria y (iv) el sector externo de la economía está equilibrado. En la práctica, no todas las estadísticas publicadas cumplen estas condiciones, por lo que resulta necesaria la realización de varios ajustes.

En la Tabla 2 se presenta una SAM que resume la construida para calibrar el Modelo General. Todas las cifras que aparecen en la tabla están expresadas en millones de dólares de 1992.

Tabla 2: Matriz de Contabilidad Social

\begin{tabular}{|lr|rrrrrr|r|}
\hline & & Firmas & Factores & Flias & Gobierno & Cuenta K & ROW & TOTAL \\
& & 1 & 2 & 3 & 4 & 5 & 6 & 7 \\
\hline Firmas & 1 & 145,880 & & 218,727 & & & 14,292 & 378,899 \\
Factores & 2 & 219,247 & & & & & & 219,247 \\
Flias & 3 & & 219,247 & & 3,043 & 5,421 & & 227,711 \\
Gobierno & 4 & 2,251 & & 793 & & & & 3,043 \\
Cuenta K & 5 & & & & & & & \\
ROW & 6 & 11,522 & & & & & & \\
TOTAL & 7 & 378,899 & 219,247 & 227,711 & 3,043 & 5,421 & 19,713 & 19,713 \\
\hline
\end{tabular}

Fuente: Elaboración propia en base a datos del GTAP, MIP 1997, SCN, EPH y TRAINS.

Para interpretar una SAM se sigue el principio de que las columnas realizan pagos a las filas. Por ejemplo, la celda $(1,2)^{20}$ expresa que los sectores productivos pagaron 219,247 millones de dólares como remuneración a los factores primarios de producción. Como muestra la celda $(3,2)$, este monto lo recibieron las familias que son dueñas de toda la dotación factorial de la economía. La celda $(1,3)$ expresa que las familias gastaron 218,727 millones de dólares en bienes que producen los sectores productivos. La celda $(4,3)$ expresa que las familias pagaron 793 millones de dólares al gobierno en concepto de aranceles a la importación. La celda $(6,3)$ expresa que las familias importaron bienes desde el resto del mundo por valor de 8,192 millones de dólares. La celda $(7,3)$ expresa que el gasto total de las familias en 1992 fue 227,711 millones de dólares. Este valor es igual al de la celda $(3,7)$ que expresa cual fue el ingreso total de las familias en ese año.

Para determinar el valor de los parámetros de participación y de escala de las funciones del modelo se emplea la información de la SAM combinada con valores (obtenidos de la literatura) de las elasticidades de sustitución y transformación. El procedimiento empleado se

\footnotetext{
${ }^{20}$ La celda (i,j) se refiere al valor correspondiente a la intersección de la fila i con la columna j.
} 
denomina calibración y consiste en obtener valores para los parámetros del modelo de manera tal que la solución inicial del modelo replique los valores de la SAM (Mansur y Whalley, 1984). Para esto, en lugar de resolver el modelo para obtener un equilibrio, se utiliza el equilibrio observado (el del caso base) para calcular los valores de los parámetros del modelo consistentes con esa observación. En la calibración se distingue entre "parámetros libres" (elasticidades) que pueden ser obtenidos de fuentes externas y "parámetros calibrados" (parámetros de distribución y escala) que son derivados de los anteriores combinados con la SAM de manera tal de reproducir el caso base.

\section{EJERCICIOS DE SIMULACION}

\section{VI.1. COMERCIO Y DESIGUALDAD SALARIAL}

Los modelos presentados más arriba pueden emplearse para calcular cuánto del cambio en el diferencial salarial se debe al aumento del comercio internacional. Para esto, la apertura comercial argentina se modela como disminuciones en las tasas arancelarias. Como se mencionara más arriba, los datos para la Argentina indican que entre 1992 y 1998, el arancel promedio cayó alrededor de 30\%. Durante el mismo período, el cociente $w_{H} / w_{L}$ pasó de 1,70 en 1992 a 1,88 en 1998, indicando un incremento de $10 \%$.

\section{RESULTADOS DEL MODELO SIMPLE}

En esta sección se presentan los resultados de realizar las simulaciones empleando el Modelo Simple. Este modelo considera que existe diferenciación de productos sólo del lado del consumo. Cuando se utiliza el Modelo Simple, se consideran dos sectores productivos: uno que es exportable neto y otro importable neto. Así, teniendo en cuenta que Argentina exporta principalmente productos intensivos en trabajo no calificado y la disminución de la tasa del arancel, se espera que el comercio tenga un efecto negativo sobre la desigualdad salarial.

En la Tabla 3 se presentan los resultados de simular la apertura comercial para distintos valores de la elasticidad de sustitución en el agregado (Armington) del bien $D$ y del bien $M$, $\varepsilon$. En todos los ejercicios, la elasticidades de sustitución entre trabajo calificado y no calificado en la producción de los bienes $E \mathrm{y} D, \Omega_{\mathrm{E}} \mathrm{y} \Omega_{\mathrm{D}}$, se mantienen iguales a $1,25^{21}$.

\footnotetext{
${ }^{21}$ Este es el valor empleado por Abrego y Whalley (1999 y 2000).
} 
Tabla 3: Resultados del Modelo Simple

\begin{tabular}{lccccc}
\hline & 0.2 & 1 & 2 & 5 & 10 \\
\hline $\begin{array}{l}\text { Porcentaje del cambio en } \\
W_{H} / W_{L} \text { debido a la } \\
\begin{array}{l}\text { apertura comercial } \\
\text { Porcentaje del cambio en }\end{array}\end{array}$ & 0.1 & -0.9 & -2.1 & -6.0 & -13.3 \\
$\begin{array}{l}W_{H} / W_{L} \text { debido a otros } \\
\text { factores }\end{array}$ & 99.9 & 100.9 & 102.1 & 106.0 & 113.3 \\
\end{tabular}

La segunda columna de la Tabla 3 indica que -cuando la elasticidad de sustitución entre $D$ y $M$ es 0,2- del aumento del diferencial salarial de 10\%, la baja de aranceles sólo puede explicar el $0,1 \%$ y el restante $99,9 \%$ es explicado por otros factores. Los resultados que se obtienen indican que el comercio no puede explicar el aumento de la desigualdad salarial ocurrido en la última década. De hecho, en este modelo, para valores plausibles de los parámetros la baja de los aranceles hace disminuir la desigualdad salarial. Se observa, además, que cuando los bienes $D$ y $M$ se hacen más sustituibles (aumenta el valor de $\varepsilon$ ) la porción del aumento en el diferencial salarial que explica el comercio disminuye.

\section{RESULTADOS MODELO GENERAL}

En esta sección se presentan los resultados obtenidos cuando se emplea el modelo más general para analizar el efecto de la apertura comercial sobre el diferencial salarial entre trabajadores con distinta calificación. Una de las ventajas de utilizar este modelo es que permite captar los cambios de las tasas arancelarias que enfrentan las importaciones de cada bien. Durante el período analizado, la baja de aranceles fue mayor para los productos manufactureros que para los productos agrícolas.

Los ejercicios de simulación se realizan utilizando distintos valores para los parámetros del modelo. El ejercicio 1 es la especificación central y los ejercicios 2, 3 y 4 constituyen un análisis de sensibilidad de ésta con respecto al valor de los parámetros más relevantes para el problema bajo estudio. En el ejercicio 1 las elasticidades de sustitución (Armington) entre bienes domésticos e importados son el doble de las reportadas en la Tabla B1 del Apéndice B y obtenidas del GTAP, la elasticidad de la frontera de transformación entre ventas al mercado doméstico y exportaciones (CET) es 8 para todos los sectores (Harrison et al., 1997) ${ }^{22}$, la

\footnotetext{
${ }^{22}$ Varios autores utilizan infinito como valor para esta elasticidad de transformación (Hertel, 1997).
} 
elasticidad de sustitución entre trabajo calificado y no calificado es 0,30 (Fallon y Layard, 1975). En todos los ejercicios las elasticidades de sustitución entre trabajo y capital son las mismas y se obtienen del GTAP. Para una descripción de las elasticidades obtenidas del GTAP véase el Apéndice B. La Tabla 4 muestra los resultados y la Tabla 5 los valores asignados a las elasticidades en cada ejercicio de simulación.

Tabla 4: Resultados del Modelo General

\begin{tabular}{lccccc}
\hline & EJ 1 & EJ 2 & EJ 3 & EJ 4 & EJ 5 \\
\hline $\begin{array}{l}\text { Porcentaje del cambio en } \\
\begin{array}{l}W_{H} / w_{L} \text { debido a la } \\
\text { apertura comercial }\end{array}\end{array}$ & 2.9 & -0.1 & 5.9 & 3.1 & 0.9 \\
$\begin{array}{l}\text { Porcentaje del cambio en } \\
W_{H} / w_{L} \text { debido a otros } \\
\text { factores }\end{array}$ & 97.1 & 100.1 & 94.1 & 96.9 & 99.1 \\
\hline
\end{tabular}

La Tabla 4 muestra que cuando se utiliza la especificación central (ejercicio 1), el aumento del comercio producto de la apertura comercial sólo explica el 2,9\% del aumento total (igual a $10 \%)$ del diferencial salarial $w_{H} / w_{L}$. El 97,1\% restante es explicado por otros factores.

Tabla 5: Valores asignados a las elasticidades

\begin{tabular}{|c|c|c|c|c|c|}
\hline ELASTICIDAD & EJ 1 & EJ 2 & EJ 3 & EJ 4 & EJ 5 \\
\hline$L$ VS. $H\left(\Omega_{\mathrm{i}}\right)$ & 0.3 & 0.3 & 0.3 & 0.3 & 1.1 \\
\hline$D$ vs. $E\left(\psi_{\mathrm{i}}\right)$ & $8 . \odot$ & $8 . \odot$ & 8.0 & $3 . \odot$ & 8.0 \\
\hline$D D$ vs. $M D \quad\left(\varepsilon_{\mathrm{i}}\right)$ & $\mathrm{GTAP}^{*} 2$ & GTAP & $\mathrm{GTAP}^{*} 3$ & $\mathrm{GTAP}^{*} 2$ & $\mathrm{GTAP} * 2$ \\
\hline$D I$ vs. $M I \quad\left(\chi_{i j}\right)$ & $\mathrm{GTAP}^{*} 2$ & GTAP & $\mathrm{GTAP}^{*} 3$ & $\mathrm{GTAP}^{*} 2$ & $\mathrm{GTAP}^{*} 2$ \\
\hline
\end{tabular}

Los resultados presentados muestran que la apertura comercial explica entre el 0 y el 6\%, aproximadamente, del aumento en el diferencial salarial ocurrido en Argentina entre 1992 y 1998. Adicionalmente, de la tabla se deduce que la porción del aumento en el diferencial salarial que explica la disminución de los aranceles es mayor cuando se aumentan las elasticidades de sustitución (Armington) entre bienes domésticos e importados. Lo mismo ocurre cuando se disminuye la elasticidad de transformación (CET) entre ventas al mercado doméstico y exportaciones al resto del mundo. Por el contrario, el efecto de la apertura comercial sobre la desigualdad salarial es menor cuando se incrementa la elasticidad de sustitución entre trabajo calificado y no calificado.

El precio doméstico de un bien (el precio de un agregado Armington) determinado es un promedio ponderado de los precios de las variedades doméstica e importada de ese bien. En 
consecuencia, mayores elasticidades Armington hacen que el sistema de precios doméstico sea más sensible a cambios en las tasas arancelarias. Por lo tanto, mayores elasticidades Armington hacen que la baja de aranceles explique más del aumento en el diferencial salarial.

La elasticidad de transformación (CET) determina la facilidad con la que cada sector productivo puede cambiar entre vender al mercado doméstico o exportar al resto del mundo. A mayor valor de esta elasticidad, la variedad que se vende al mercado doméstico y la variedad que se exporta al resto del mundo son más similares por lo que el precio de la variedad doméstica es más sensible a las variaciones en el precio (exógeno) de la variedad que se exporta. En consecuencia, un aumento en el valor de la elasticidad de transformación hace que el precio de la variedad doméstica responda con menor intensidad a cambios en el precio de la variedad importada (a través del agregado Armington) ya que si parte de la producción de la variedad doméstica no se vende, puede más fácilmente transformarse en exportaciones al resto del mundo. Por lo tanto, una menor elasticidad de transformación hace que la baja de aranceles explique una porción mayor del aumento en el diferencial salarial.

Adicionalmente, en el Apéndice $C$ se presentan los resultados de simular la apertura comercial argentina pero diferenciando por tipo de importación: bienes de capital, insumos intermedios y bienes para consumo final.

\section{VI.2. CALCULO DEL CAMBIO TECNOLOGICO}

El objetivo de realizar este cálculo es únicamente metodológico ya que los cambios ocurridos en la economía argentina durante los noventa hacen imposible considerar que el único factor, además de la apertura comercial, que explica el aumento de la desigualdad salarial es el cambio tecnológico sesgado contra los trabajadores no calificados. Esta metodología se basa en la propuesta por Abrego y Whalley (1999 y 2000) y puede ser empleada para realizar distintos ejercicios de descomposición con un modelo de CGE.

Debido a que el cambio tecnológico es el factor que aparece en la literatura como causa principal del aumento de la desigualdad salarial, luego de calculado el efecto de la apertura comercial sobre el aumento del diferencial salarial, se realiza un ejercicio en el que se determina -de manera residual- cuál es el cambio tecnológico necesario para que el modelo de como solución -cuando se introducen simultáneamente ambos cambios de manera exógenaun cambio en el cociente $w_{H} / w_{L}$ igual al ocurrido en Argentina en el período 1992 a 1998. 
El tipo de cambio tecnológico que se considera es específico del factor trabajo. En particular, es sesgado contra el trabajo no calificado. El cambio tecnológico se modela como variaciones en los parámetros de participación de las funciones de producción de bienes. Debido a que los parámetros de participación en cada función de producción suman uno, un shock adverso al trabajo no calificado disminuye el parámetro de participación de $L$ en relación al de $H$ para cada sector productivo.

A los fines de descomponer el aumento observado en la desigualdad salarial en un componente atribuible al comercio internacional y otro al cambio tecnológico, el modelo se resuelve cinco veces. En la Tabla D1 del Apéndice D se indica cuáles son las variables exógenas y endógenas en cada corrida del Modelo Simple. Para los ejercicios de descomposición del cambio en $w_{H} / w_{L}$, en primer lugar se determina el cambio en el diferencial salarial producto del cambio en los aranceles, en segundo lugar, se obtiene (resolviendo un modelo donde el cambio tecnológico es una variable endógena y el diferencial salarial está dado exógenamente) el cambio tecnológico necesario para que el diferencial salarial sea igual al observado (en el caso argentino, 10\% en el período 1992 a 1998). El resultado de cada uno de los ejercicios de descomposición que se realizan depende de los valores que tomen los parámetros libres del modelo (elasticidades en la producción y en el consumo).

\section{CONCLUSIONES Y EXTENSIONES}

- Del análisis realizado en este trabajo se desprende que la apertura comercial no es una causa importante del aumento de la desigualdad salarial ocurrido durante la década del noventa. Utilizando un modelo de CGE con una estructura estándar se obtuvo que, para valores usuales de los parámetros del modelo, el comercio explica alrededor del 3\% del aumento en el diferencial salarial ocurrido en Argentina durante la década del noventa. Este resultado es bajo cuando se los compara con las estimaciones presentes en la literatura.

- La comparación de los resultados obtenidos con el Modelo Simple y con el Modelo General permiten apreciar la importancia que tienen la estructura y la dimensión del modelo para obtener un resultado donde el comercio explica parte del aumento del diferencial salarial. 
- Los modelos de CGE son ampliamente utilizados para el análisis cuantitativo de políticas. Sin embargo, la metodología del CGE es frecuentemente criticada. Los aspectos más criticados son la utilización de valores aparentemente arbitrarios para los parámetros de comportamiento (elasticidades) y la falta de validación empírica de la estructura de los modelos. Estas deficiencias son particularmente problemáticas si se tiene en cuenta que los resultados del modelo son usualmente muy sensibles a estos supuestos. En el caso de las elasticidades de sustitución Armington utilizadas en este trabajo, en un trabajo reciente, Liu et al. (2001) demuestran, en el contexto del modelo estándar del GTAP ${ }^{23}$, que los valores de las elasticidades Armington de la base de datos del GTAP utilizadas en este trabajo no resultan marcadamente diferentes de las que estiman como correctas en un ejercicio de predicción hacia atrás ${ }^{24}$.

- Se presentó una metodología que puede ser empleada para realizar ejercicios de descomposición con un modelo de CGE.

- El modelo utilizado en este trabajo posee una estructura muy simple. Además se trata de un modelo que sólo capta los efectos estáticos de la baja arancelaria. Por lo tanto, una posible extensión del presente trabajo es la inclusión de aspectos no estándar en el modelo de CGE como por ejemplo, la acumulación de capital (Francois y Reinert, 1997), externalidades debidas al comercio (De Melo y Robinson, 1992), costos de transacción (Bussolo, 2001), economías de escala y competencia imperfecta (Francois y Reinert, 1997), etc. Adicionalmente, también podría incorporarse al modelo el sector financiero de la economía. En el caso argentino, esto es particularmente importante ya que, durante los noventa, además de la apertura comercial se produjo una apertura financiera.

- Otra posible extensión es la utilización de un modelo de CGE multiregional que permita diferenciar el efecto sobre la desigualdad salarial de la apertura comercial unilateral y del Mercosur. En este sentido, una posibilidad es realizar simulaciones de "predicción hacia atrás” utilizando la versión 5 de la base de datos del GTAP (que contiene información referida al año 1997) y un modelo multiregional similar al presentado por Rutherford y Paltsev (2000).

\footnotetext{
${ }^{23}$ El modelo estándar del GTAP es un modelo de CGE multisectorial y multiregional ampliamente utilizado para el análisis de políticas comerciales desde una perspectiva global.

${ }^{24}$ Sin embargo, rechazan la hipótesis de igualdad entre las elasticidades estándar del GTAP y las estimadas.
} 
- Teniendo en cuenta que en este trabajo se simuló la apertura comercial únicamente como una baja de aranceles, es probable que los resultados obtenidos subestimen el efecto del comercio sobre el diferencial salarial ya que la apertura comercial argentina se llevó a cabo también a través de la eliminación de restricciones cuantitativas ${ }^{25}$.

\footnotetext{
${ }^{25}$ Para ver la importancia de las medidas de protección no arancelarias puede consultarse Bouët et al. (2001).
} 


\section{BIBLIOGRAFIA}

Abdelkhalek, Touhami and Dufour, Jean-Marie (1998). Statistical Inference for Computable General Equilibrium Models, with Application to a Model of the Moroccan Economy. The Review of Economics and Statistics 80 (4): 520-534.

Abdelkhalek, Touhami and Dufour, Jean-Marie (2000). Confidence Regions for Calibrated Parameters in Computable General Equilibrium Models. Cirano Scientific Series 2000s-18.

Abrego, Lisandro and Whalley, John (1999). The Choice of Structural Model in Trade-Wages Decompositions. NBER Working Paper 7312.

Abrego, Lisandro and Whalley, John (2000). Demand Side Considerations and the Trade and Wages Debate. NBER Working Paper 7674.

Armington, Paul S. (1969). A Theory of Demand for Products Distinguished by Place of Production. International Monetary Fund Staff Papers 16: 159-178.

Arrow, K. J. and Debreu, G. (1954). Existence of an Equilibrium for a Competitive Economy. Econometrica 22: 265-290.

Arrow, Kenneth J. and Kehoe, Timothy J. (1994). Herbert Scarf's Contributions to Economics. Journal of Economis Perspectives 8 (4): 161-181.

Autor, David H., Katz, Lawrence F. and Krueger, Alan B. (1998). Computing Inequality: Have Computers Changed the Labor Market?. The Quarterly Journal of Economics 113 (4): 1169-1213.

Baldwin, Robet E. and Cain Glen G. (1997). Shifts in U.S. Relative Wages: The Role of Trade, Technology and Factor Endowments. NBER Working Paper 5934.

Bebczuk, Ricardo and Gasparini, Leonardo (2001). Globalisation and Inequality: The Case of Argentina. Departamento de Economía de la Universidad Nacional de La Plata Documento de Trabajo 32.

Berlinski, Julio (1998). El Sistema de Incentivos en Argentina (De la Liberalización Unilateral al Mercosur). Instituto Torcuato Di Tella Documento de Trabajo.

Berrettoni, Daniel y Cicowiez, Martín (2001). La Economía Bonaerense Frente al Proceso de Integración Continental: Posibles Impactos de la Conformación del ALCA. Maestría en Finanazas Públicas de la Universidad Nacional de La Plata.

Bhattarai, Keshab; Ghosh, Madanmohan and Whalley, John (1998). More on Trade Closure.

Bhattarai, Keshab; Ghosh, Madanmohan and Whalley, John (1999). On Some Properties of a Trade Closure Widely Used in Numerical Modelling. Economic Letters 62: 13-21.

Bouët, Antoine; Fontagne, Lionel; Mimouni, Mondher and Pichot, Xavier (2001). Market Access Maps: A Bilateral and Disaggregated Measure of Market Access. Conference on Impacts of Trade Liberalization Agreements on Latin America and the Caribbean. 
Bound, John and Johnson, George (1992). Changes in the Structure of Wages in the 1980's: An Evaluation of Alternative Explanations. The American Economic Review 82 (3): 371-392.

Brooke, A.; Kendrick, D. and Meeraus, A. (1996). GAMS Release 2.25: A user's guide. GAMS Development Corporation.

Burtless, Gary (1995). International Trade and the Rise in Earnings Inequality. Journal of Economic Literature 33: 800-816.

Bussolo, Maurizio (2001). How Many Forms Do I Have to Fill In to Export My Coffee? The Role of Transaction Costs in Explaining Economic Performance in Latin America. Conference on Impacts of Trade Liberalization Agreements on Latin America and the Caribbean.

Card, David (1998). Falling Union Membership and Rising Wage Inequality: What's the Connection? NBER Working Paper 6520.

Chisari, Omar y Romero, Carlos (1996). Distribución del Ingreso, Asignación de Recursos y Shocks Macroeconómicos: Un Modelo de Equilibrio General Computado para la Argentina en 1993. CEPAL.

Cristini, Marcela (1999). Apertura Económica, Política Comercial y la Distribución del Ingreso: ¿Qué Aporta el Caso Argentino al Debate?. En FIEL, La Distribución del Ingreso en Argentina.

Melo de, Jaime (1988). Computable General Equilibrium Models for Trade Policy Analysis in Developing Countries: A Survey. Jounal of Policy Modeling 10 (4): 469-503.

Melo de, Jaime and Robinson, Sherman (1989). Product Differentiation and The Treatment of Foreign Trade in Computable General Equilibrium Models of Small Economies. Journal of International Economics 27: 47-67.

Melo de, Jaime and Robinson, Sherman (1990). Productivity and Externalities: Models of Export-Led Growth. The World Bank Working Papers.

Diao, Xinshen; Díaz-Bonilla Eugenio and Robinson, Sherman (2001). Scenarios for Trade Integration in the Americas. Conference on Impacts of Trade Liberalization Agreements on Latin America and the Caribbean.

Fallon, P. R. and Layard, P. R. G. (1975). Capital-Skill Complementarity, Income Distribution, and Output Accounting. Journal of Political Economy 83 (2): 279-301.

Feenstra, Robert C. and Hanson, Gordon H. (1996). Globalization, Outsourcing, and Wage Inequality. The American Economic Review 86 (2): 240-245.

Feenstra, Robert C. and Hanson, Gordon H. (1999). The Impact of Outsourcing and HighTechnology Capital on Wages: Estimates for the United States, 1979-1990. The Quarterly Journal of Economics 114 (3): 907-940.

Francois, Joseph F. and Nelson, Doulas (1998). Trade, Technology, and Wages: General Equilibrium Mechanics. The Economic Journal 108: 1483-1499 
Francois, Joseph F. and Reinert, Kenneth A. (eds.) (1997). Applied Methods for Trade Policy Analysis: A Handbook. Cambridge: Cambridge University Press.

Gasparini, Leonardo; Marchionni, Mariana y Sosa Escudero, Walter (2001). Distribución del Ingreso en la Argentina. Fundación Arcor.

Gehlhar, Mark J. (1997). Historical Analysis of Growth and Trade Patterns in the Pacific Rim: An Evaluation of the GTAP Framework. In Hertel, Thomas W. (ed.). Global Trade Analysis: Modeling and Applications. Cambridge: Cambridge University Press.

Ginsburgh, Victor and Keyzer, Michiel (1997). The Structure of Applied General Equilibrium Models. MIT Press.

Harberger, A. C. (1962). The Incidence of the Corporation Income Tax. Journal of Political Economy 70: 215-240.

Harrison, Glenn W.; Rutherford, Thomas F. and Tarr, David G. (1997). Trade Policy Options for Chile: A Quantitative Evaluation. The World Bank Working Papers 1783.

Haskel, Jonathan and Slaughter, Matthew J. (1999). Trade, Technology and U.K. Wage Inequality. NBER Working Paper 6978.

Hertel, Thomas W. (ed.) (1997). Global Trade Analysis: Modeling and Applications. Cambridge: Cambridge University Press.

Hillberry, Russell; Anderson, Michael; Balisteri, Edward and Fox, Alan (2001). The Determinants of Armington Taste Parameters in CGE Models, or "Why You Love Canadian Vegetable Oil”. Fourth Annual Conference on Global Economic Analysis.

Johnson, Harry G. (1966). Factor Market Distortions and the Shape of the Transformation Curve. Econometrica 34 (3): 686-698

Katz, Lawrence F. and Murphy, Kevin M. (1992). Changes in Relative Wages, 1963-1987: Supply and Demand Factors. The Quarterly Journal of Economics 107 (1): 35-78.

Kehoe, Patrick J. and Kehoe, Timothy J. (1994). A Primer on Static Applied General Equilibrium Models. Federal Reserve Bank of Minneapolis Quarterly Review 18 (1).

Kehoe, Timothy J. (1996). Social Accounting Matrices and Applied General Equilibrium Models. Federal Reserve Bank of Minneapolis Working Paper 563.

Krugman, Paul R. (2000). Technology, Trade and Factor Prices. Journal of International Economics 50 (1): 51-71.

Liu, Jing; Arndt, Channing and Hertel, Thomas (2001). Parameter Estimation and Measures of FIT in A Global General Equilibrium Model. Fourth Annual Conference on Global Economic Analysis.

Malcolm, Gerard (1998). Adjusting Tax Rates in the GTAP Data Base. GTAP Technical Paper 12. 
Mansur, A. H. and Whalley, J. (1984). Numerical Specification of Applied General Equilibrium Models: Estimation, Calibration and Data. In Scarf, H. E. and Shoven, J. B. (eds.). Applied General equilibrium Analysis. Cambridge: Cambridge University Press.

Marquez, Jaime (1994). The Econometrics of Elasticities or the Elasticity of Econometrics: An Empirical Analysis of the Behavior of U.S. Imports. The Review of Economics and Statistics 76 (3): 471-481.

McDougall, R. A.; Elbehri, A. and Truong, T. P. (1998). Global Trade Assistance and Protection: The GTAP 3 Data Base. Center for Global Trade Analysis, Purdue University.

Porto, Guido (2000). Comercio Internacional y Desigualdad Laboral. Departamento de Economía de la Uiversidad Nacional de La Plata Documento de Trabajo.

Richardson, J. David (1995). Income Inequality and Trade: How to Think, What to Conclude. Journal of Economic Perspectives 9 (3): 33-55.

Rutherford, Thomas F. (1999). Applied General Equilibrium Modeling with MPSGE as a GAMS Subsystem: An Overview of the Modeling Framework and Syntax. Computational Economics 14: 1-46.

Rutherford, Thomas F. and Paltsev, Segey V. (2000). GTAPinGAMS and GTAP-EG: Global Datasets for Economic Research and Illustrative Models. Department of Economics University of Colorado Working Paper.

Scarf, Herbert (1969). An Example of an Algorithm for Calculating General Equilibrium Prices. American Economic Review 59: 669-677.

Shoven, John B. and Whalley John (1972). A General Equilibrium Calculation of the Effects of Differential Taxation of Income from Capital in the U. S. Journal of Public Economics 1: 281-321.

Shoven, John B. and Whalley, John (1992). Applying general equilibrium. Cambridge: Cambridge University Press.

Shoven, John B. and Whalley, John (1984). Applied General-Equilibrium Models of Taxation and International Trade: An Introduction and Survey. Journal of Economic Literature 22: 1007-1051.

St-Hilaire, France and Whalley, John (1983). A Microconsistent Equilibrium Data Set for Canada for Use in Tax Policy Analysis. Review of Income and Wealth 29: 175-204.

Whalley, John and Yeung, Bernard (1984). External Sector 'Closing' Rules in Applied General Equilibrium Models. Journal of International Economics 16: 123-138.

Wood, Adrian (1995). How Trade Hurt Unskilled Workers. Journal of Economic Perspectives 9 (3): 57-80.

Wood, Adrian (1997). Openness and Wage Inequality in Developing Countries: The Latin American Challenge to East Asian Conventional Wisdom. The World Bank Economic Review 11 (1): 33-57. 


\section{APENDICE A: ECUACIONES DEL MODELO GENERAL}

En este apéndice se presenta, de manera detallada, la estructura del Modelo General utilizado en los ejercicios de simulación. Las ecuaciones se expresan de manera análoga a como fueron codificadas en GAMS. Se trata de un sistema de ecuaciones no lineales de 537x537. Los subíndices i y j se refieren a los bienes considerados en el modelo. Es decir, $i=j=\left\{G_{-} L\right.$, OAF, FIN, TEX, NRM, MPC, TRN, C_O, M_M, SVC\}.

\section{A.1. PARAMETROS}

\section{ELASTICIDADES}

$\xi_{i} \quad$ Elasticidad de sustitución entre el agregado de trabajo y el capital

$\Omega_{i} \quad$ Elasticidad de sustitución entre trabajo calificado y no calificado

$\sigma_{i} \quad$ Elasticidad de sustitución en el consumo final (nivel superior)

$\psi_{i} \quad$ Elasticidad de transformación (CET) entre ventas al mercado doméstico y exportaciones

$\varepsilon_{i} \quad$ Elasticidad de sustitución (Armington) entre bienes domésticos e importados en el consumo final

$\chi_{j i} \quad$ Elasticidad de sustitución (Armington) entre bienes domésticos e importados en el consumo intermedio

\section{PARAMETROS DE DISTRIBUCION Y ESCALA}

$\imath_{j i}^{D} \quad$ Participación de bienes domésticos en el consumo intermedio (Armington)

$\imath_{j i}^{M} \quad$ Participación de bienes importados en el consumo intermedio (Armington)

$\zeta_{j i} \quad$ Parámetro de escala en el consumo intermedio (Armington)

$\delta_{i} \quad$ Participación del trabajo no calificado en el agregado de trabajo

$\phi_{i} \quad$ Parámetro de escala en el agregado de trabajo

$v_{i}^{N} \quad$ Participación del agregado de trabajo en la producción de valor agregado

$v_{i}^{K} \quad$ Participación del capital en la producción de valor agregado 
$\mu_{i} \quad$ Parámetro de escala en la producción de valor agregado

$\eta_{i}^{D} \quad$ Participación de ventas al mercado doméstico en ventas totales

$\eta_{i}^{E} \quad$ Participación de exportaciones en ventas totales

$\theta_{i} \quad$ Parámetro de escala en la función de transformación (CET)

$\alpha_{i} \quad$ Participación de i en el consumo final

$\beta_{i}^{D} \quad$ Participación de bienes domésticos en el consumo final (Armington)

$\beta_{i}^{M} \quad$ Participación de bienes importados en el consumo final (Armington)

$\gamma_{i} \quad$ Parámetro de escala en el consumo final (Armington)

\section{OTROS}

$\bar{K} \quad$ Dotación de capital

$\bar{L} \quad$ Dotación de trabajo no calificado

$\bar{H} \quad$ Dotación de trabajo calificado

$I_{j i} \quad$ Requerimiento de insumo j por unidad de producto i

$\bar{B} \quad$ Déficit comercial en el equilibrio inicial

$\overline{P_{i}^{M}} \quad$ Precio mundial de las importaciones de i

$\overline{P_{i}^{E}} \quad$ Precio mundial de las exportaciones de i

$\tau_{j i}^{I} \quad$ Tasa del arancel al consumo intermedio de j en la producción de i

$\tau_{i}^{D} \quad$ Tasa del arancel al consumo final de i

PREMIO Diferencial salarial

\section{A.2. VARIABLES}

$A I_{j i} \quad$ Consumo intermedio de j en la producción de i

$D I_{j i} \quad$ Consumo intermedio de j doméstico en la producción de i

MI $I_{j i} \quad$ Consumo intermedio de j importado en la producción de i 


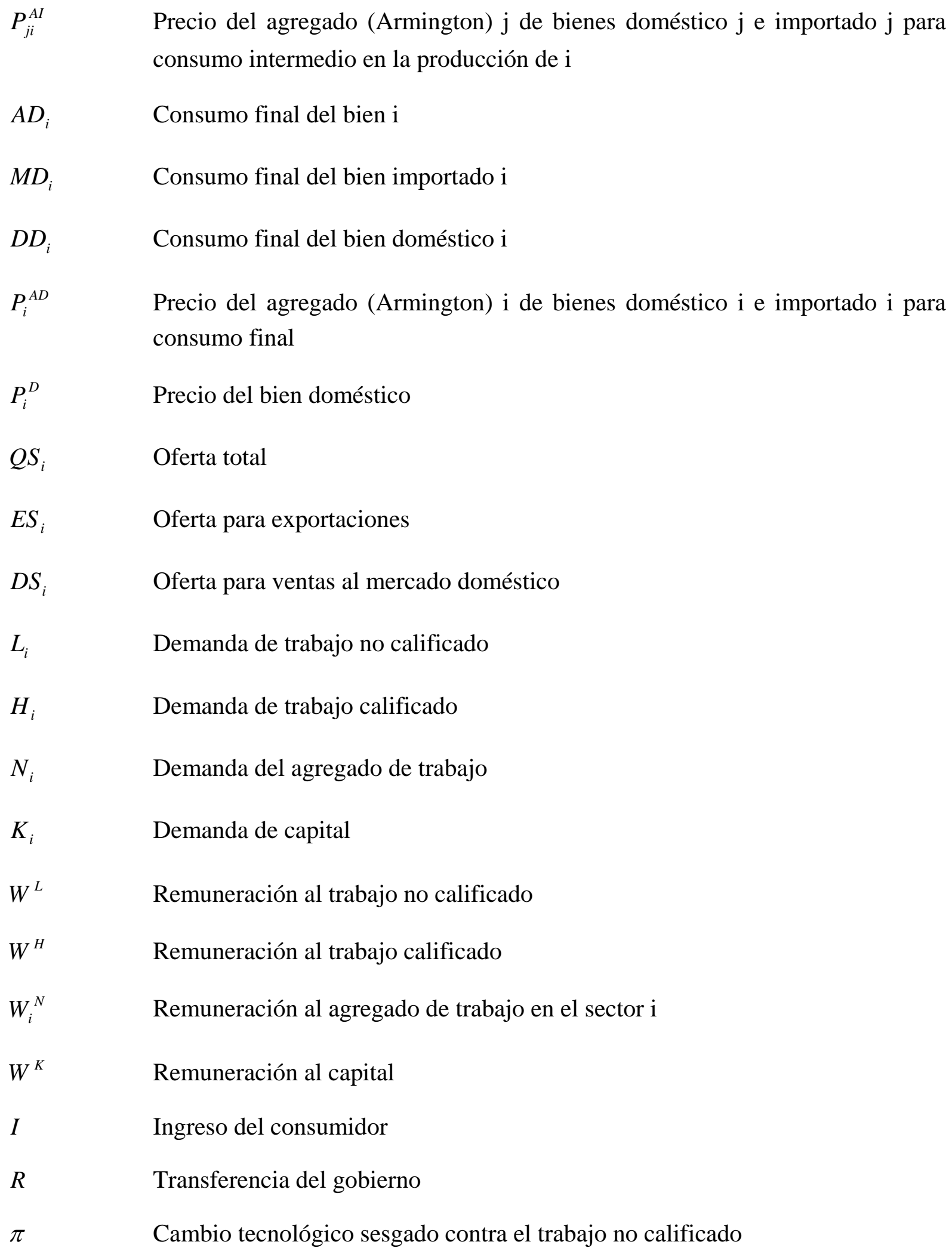

\section{A.3. ECUACIONES}

Para facilitar la lectura, las ecuaciones del modelo fueron agrupadas en seis bloques. En las secciones siguientes se presentan las ecuaciones pertenecientes a cada uno de ellos. 


\section{BLOQUE DE OFERTA DE BIENES}

Las ecuaciones de este bloque surgen de la maximización de beneficios del productor. Cada productor maximiza sus beneficios sujeto a una función de transformación tipo CET. Esta función de transformación determina la cantidad que vende en el mercado doméstico y la cantidad que exporta al resto del mundo. La ecuación (1) es la función de oferta de bienes al mercado doméstico y la ecuación (2) es la función de oferta de exportaciones al resto del mundo. La ecuación (3) es la condición de cero beneficios del productor.

$$
\begin{aligned}
& D S_{i}=\frac{\left(\eta_{i}^{D}\right)^{-\psi_{i}}\left(P_{i}^{D}\right)^{\psi_{i}} Q S_{i}}{\theta_{i}\left[\left(P_{i}^{D}\right)^{\left(1+\psi_{i}\right)}\left(\eta_{i}^{D}\right)^{-\psi i}+\left(\overline{P_{i}^{E}}\right)^{\left(1+\psi_{i}\right)}\left(\eta_{i}^{E}\right)^{-\psi i}\right]^{\frac{\psi_{i}}{1+\psi_{i}}}} \\
& E S_{i}=\frac{\left(\eta_{i}^{E}\right)^{-\psi_{i}}\left(\overline{P_{i}^{E}}\right)^{\psi_{i}} Q S_{i}}{\left.\left.\theta_{i}\left[\left(P_{i}^{D}\right)^{\left(1+\psi_{i}\right.}\right)\left(\eta_{i}^{D}\right)^{-\psi i}+\left(\overline{P_{i}^{E}}\right)^{\left(1+\psi_{i}\right.}\right)\left(\eta_{i}^{E}\right)^{-\psi i}\right]^{\frac{\psi_{i}}{1+\psi_{i}}}} \\
& \overline{P_{i}^{E}} E S_{i}+P_{i}^{D} D S_{i}=W_{i}^{N} N_{i}+W^{K} K_{i}+\sum_{j} P_{j i}^{A I} A I_{j i}
\end{aligned}
$$

\section{BLOQUE DE DEMANDA DE FACTORES PRIMARIOS}

Las ecuaciones de este bloque surgen de la minimización de costos de los productores. En primer lugar, cada productor combina, en proporciones fijas, valor agregado e insumos intermedios. Por el lado del valor agregado, la ecuación (4) es la función de demanda del agregado de trabajo $(N)$ y la ecuación (5) es la función de demanda de capital $(K)$. En la siguiente etapa, el productor determina la composición de trabajo no calificado $(L)$, ecuación (6), y trabajo calificado $(H)$, ecuación (7), del trabajo total que demanda. Por último, la ecuación (8) asegura que el gasto total en el factor trabajo se distribuya entre pagos a las dos variedades de trabajo.

$$
\begin{aligned}
& N_{i}=\frac{\left(v_{i}^{N}\right)^{\xi_{i}} Q S_{i}}{\mu_{i}\left(W^{N}\right)^{\xi_{i}}\left[\left(v_{i}^{K}\right)^{\xi_{i}}\left(W^{K}\right)^{\left(1-\xi_{i}\right)}+v_{i}^{N} \xi_{i}\left(W_{i}^{N}\right)^{\left(1-\xi_{i}\right)}\right]^{\xi_{i}-1}} \\
& K_{i}=\frac{\left(v_{i}^{K}\right)^{\xi_{i}} Q S_{i}}{\mu_{i}\left(W^{K}\right)^{\xi_{i}}\left[\left(v_{i}^{K}\right)^{\xi_{i}}\left(W^{K}\right)^{\left(1-\xi_{i}\right)}+v_{i}^{N} \xi_{i}\left(W_{i}^{N}\right)^{\left(1-\xi_{i}\right)}\right]^{\xi_{i}-1}}
\end{aligned}
$$




$$
\begin{aligned}
& L_{i}=\frac{\left(\delta_{i} \pi\right)^{\Omega_{i}} N}{\phi_{i}\left(W^{L}\right)^{\Omega_{i}}\left[\left(\delta_{i} \pi\right)^{\Omega_{i}}\left(W^{L}\right)^{\left(1-\Omega_{i}\right)}+\left(1-\delta_{i} \pi\right)^{\Omega_{i}} W^{H\left(1-\Omega_{i}\right)}\right]^{\Omega_{i}-1}} \\
& H_{i}=\frac{\left[\left(1-\delta_{i} \pi\right)\right]^{\Omega_{i}} N}{\phi_{i}\left(W^{H}\right)^{\Omega_{i}}\left[\left(\delta_{i} \pi\right)^{\Omega_{i}}\left(W^{L}\right)^{\left(1-\Omega_{i}\right)}+\left(1-\delta_{i} \pi\right)^{\Omega_{i}} W^{H\left(1-\Omega_{i}\right)}\right]^{\Omega_{i}-1}} \\
& W_{i}^{N} N=W^{L} L_{i}+W^{H} H
\end{aligned}
$$

\section{BLOQUE DE DEMANDA DE INSUMOS INTERMEDIOS}

Las ecuaciones (9), (10) y (11) definen el agregado Armington de insumos intermedios. La ecuación (9) es la función de demanda intermedia de bienes domésticos y la ecuación (10) es la función de demanda intermedia de bienes importados. La ecuación (11) es la condición de cero beneficios en la producción de este agregado Armington. Por último, la ecuación (12) determina la cantidad demandada de insumo intermedio j por unidad de bien i. El lado derecho de esta ecuación es la función de producción de valor agregado que utiliza como insumos el agregado de trabajo $(N)$ y capital $(K)$.

$$
\begin{aligned}
& D I_{j i}=\frac{\left(l_{j i}^{D}\right)^{\chi_{j i}} A I_{j i}}{\zeta_{j i}\left(P_{i}^{D}\right)^{\chi_{j i}}\left\{\left(l_{j i}^{M}\right)^{\chi_{j i}}\left[\overline{P_{i}^{M}}\left(1+\tau_{j i}^{I}\right)\right]^{\left(1-\chi_{j i}\right)}+\left(l_{j i}^{D}\right)^{\chi_{j i}} P_{i}^{\left.D^{\left(1-\chi_{j i}\right.}\right)}\right) \frac{\chi_{j i}^{\chi_{j i}-1}}{x^{2}}} \\
& M I_{j i}=\frac{\left(l_{j i}^{M}\right)^{\chi_{j i}} A I_{j i}}{\zeta_{j i}\left(\overline{P_{i}^{M}}\right)^{\chi_{j i}}\left(\left(l_{j i}^{M}\right)^{\chi_{j i}}\left[\overline{P_{i}^{M}}\left(1+\tau_{j i}^{I}\right)\right]^{\left(1-\chi_{j i}\right)}+\left(l_{j i}^{D}\right)^{\chi_{j i}} P_{i}^{D\left(1-\chi_{j i}\right)}\right) \frac{\chi_{j i}}{\chi_{j i}-1}} \\
& P_{j i}^{A I} A I_{j i}=\overline{P_{i}^{M}}\left(1+\tau_{j i}^{I}\right) M I_{j i}+P_{i}^{D} D I_{j i} \\
& \frac{A I_{j i}}{I O_{j i}}=\mu_{i}\left[v_{i}^{N} N_{i}^{\frac{\xi_{i}-1}{\xi_{i}}}+v_{i}^{K} K_{i}^{\frac{\xi_{i}-1}{\xi_{i}}}\right]^{\frac{\xi_{i}}{\xi_{i}-1}}
\end{aligned}
$$

\section{BLOQUE DE DEMANDA FINAL}

Las ecuaciones de este bloque surgen de la maximización de la utilidad del consumidor representativo. Esta maximización de utilidad se realiza en dos etapas. En la primera, el consumidor determina cuánto consume de cada bien, ecuación (13). En la segunda, determina cuánto de la variedad doméstica y cuánto de la variedad importada de cada bien consume, 
ecuaciones (14) y (15), respectivamente. Por último, la ecuación (16) asegura que el gasto total del consumidor se distribuya completamente entre bienes domésticos e importados.

$$
\begin{aligned}
& A D_{i}=\frac{\alpha_{i} I}{\left(P_{i}^{A D}\right)^{\sigma} \sum_{j} \alpha_{j}\left(P_{j}^{A D}\right)^{(1-\sigma)}} \\
& D D_{i}=\frac{\left(\beta_{i}^{D}\right)^{\varepsilon_{i}} A D_{i}}{\left.\gamma_{i}\left(P_{i}^{D}\right)^{\varepsilon_{i}}\left\{\left(\beta_{i}^{D}\right)^{\varepsilon_{i}} P_{i}^{D^{\left(1-\varepsilon_{i}\right)}}+\left(\beta_{i}^{M}\right)^{\varepsilon_{i}}\left[\overline{P_{i}^{M}}\left(1+\tau_{i}^{D}\right)\right]\right]^{\left(1-\varepsilon_{i}\right)}\right\}{\frac{\varepsilon_{i}}{\varepsilon_{i}-1}}^{\left(\beta_{i}^{M}\right)^{\varepsilon_{i}} A D_{i}}} \\
& M D_{i}=\frac{\gamma_{i}\left(\overline{P_{i}^{M}}\right)^{\varepsilon_{i}}\left\{\left(\beta_{i}^{D}\right)^{\varepsilon_{i}} P_{i}^{\left.D^{\left(1-\varepsilon_{i}\right.}\right)}+\left(\beta_{i}^{M}\right)^{\varepsilon_{i}}\left[\overline{P_{i}^{M}}\left(1+\tau_{i}^{D}\right)\right]\left(1-\varepsilon_{i}\right)\right) \frac{\varepsilon_{i}}{\varepsilon_{i}-1}}{P_{i}^{A D} A D_{i}=\overline{P_{i}^{M}}\left(1+\tau_{i}^{D}\right) M D_{i}+P_{i}^{D} D D_{i}}
\end{aligned}
$$

\section{BLOQUE DE CONDICIONES DE EQUILIBRIO}

Este bloque contiene las condiciones de equilibrio en los distintos mercados del modelo. La ecuación (17) es la condición de equilibrio en el mercado del bien doméstico i. Las ecuaciones (18), (19) y (20) son las condiciones de equilibrio en los mercados de $L, H$ y $K$, respectivamente. La ecuación (21) es la restricción presupuestaria del agente representativo que expresa que el ingreso total del consumidor es igual a la remuneración a los factores productivos más la transferencia que recibe del gobierno más la transferencia que recibe desde el resto del mundo (exógena e igual al saldo de la balanza comercial). La ecuación (22) es la restricción presupuestaria del gobierno que indica que la transferencia que realiza el gobierno es igual al monto recaudado por el arancel. Finalmente, la ecuación (23) es la condición de equilibrio del sector externo de la economía.

$$
\begin{aligned}
& D S_{i}=D D_{i}+\sum_{j} I D_{i j} \\
& \bar{L}=\sum_{i} L_{i} \\
& \bar{H}=\sum_{i} H_{i} \\
& \bar{K}=\sum_{i} K_{i} \\
& I=W^{H} \bar{H}+W^{L} \bar{L}+W^{K} \bar{K}+R+\bar{B}
\end{aligned}
$$




$$
\begin{aligned}
& \sum_{i}\left(\overline{P_{i}^{M}} \tau_{i}^{D} M D_{i}+\sum_{j} \overline{P_{i}^{M}} \tau_{i j}^{I} M I_{i j}\right)=R \\
& \sum_{i} P_{i}^{E} E S_{i}+\bar{B}=\sum_{i}\left(P_{i}^{M} M D+\sum_{j} P_{i}^{M} M I_{i j}\right)
\end{aligned}
$$

\section{BLOQUE PARA ESTIMAR EL CAMBIO TECNOLOGICO}

La ecuación (24) forma parte de la estructura del modelo cuando se lo resuelve para obtener el cambio necesario en la variable que representa el cambio tecnológico $(\pi)$ para que el modelo replique el cambio en el diferencial salarial observado en el período 1992 a 1998.

$$
W^{H}=(P R E M I O) W^{L}
$$

El cambio tecnológico se modela como una variación en el valor del parámetro $\delta_{\mathrm{i}}$ de las funciones de producción de bienes. Cuando el cambio tecnológico es sesgado a favor de la mano de obra calificada, el valor del parámetro $\delta_{\mathrm{i}}$ disminuirá en una fracción común en todos los sectores productivos calculada como residuo luego de introducido el cambio en las tasas arancelarias.

En las ecuaciones referidas al lado de la producción del modelo, el parámetro $\delta_{\mathrm{i}}$ aparece multiplicado por una variable $\pi$. Para replicar la SAM, $\pi$ se fija igual a uno. Luego, cuando se introducen los cambios en las tasas arancelarias se agrega una ecuación que fija el diferencial salarial en $10 \%\left(w_{H}=1.10 w_{L}\right)$ y el valor de la variable $\pi$ se determina endógenamente cuando se soluciona el modelo.

\section{A.4. CALCULO DEL EQUILIBRIO}

Para computar cada solución del modelo, se debe resolver el sistema de ecuaciones no lineales presentado más arriba. Empleando GAMS/MINOS, esto se puede realizar maximizando una función constante sujeta a restricciones que representan el modelo. 


\section{APENDICE B: MATRIZ DE CONTABILIDAD SOCIAL Y ELASTICIDADES}

\section{B.1. MATRIZ DE CONTABILIDAD SOCIAL}

En este apéndice se describen las fuentes de información utilizadas para construir la SAM empleada para calibrar el modelo de CGE. Además, se describen los ajustes realizados a la información original.

La fuente de información principal para la confección de la SAM es la versión 3 de la base de datos del Global Trade Analysis Project (GTAP) que contiene información referida al año 1992. Esta base de datos se encuentra extensamente documentada en Hertel (1997) y McDougall et al. (1998). El GTAP es un consorcio integrado por organizaciones gubernamentales y de investigación que funciona en la Universidad Purdue de Estados Unidos $^{26}$. Uno de los objetivos básicos del GTAP es facilitar la realización de análisis de CGE sobre cuestiones relacionadas (principalmente) con el comercio internacional. La base de datos del GTAP es ampliamente utilizada por académicos y agencias gubernamentales de varios países del mundo para la realización de trabajos sobre comercio internacional que utilizan modelos de CGE. Esta base de datos está formada por Matrices de Contabilidad Social para los principales países del mundo y para varias regiones agregadas y por los flujos de comercio entre estos países o regiones. Toda la información en esta base de datos está referida a un año particular que se toma como base y todos los datos se encuentran expresados en base a una nomenclatura común.

Del GTAP se extrajo información sobre estructura productiva y flujos de comercio para la Argentina. La información obtenida del GTAP se complementó con información de la EPH, la MIP argentina del año 1997 e información sobre tasas arancelarias obtenida de TRAINS ${ }^{27}$.

Una de las características más relevantes para este trabajo de la SAM utilizada es el procedimiento que se empleó para determinar el uso de trabajo calificado y trabajo no calificado en cada sector productivo. Al respecto, se siguió el trabajo "Disaggregating Labor Payments by Skill Level in GTAP” de Jing Liu, Nico van Leeuwen, Tri Thanh Vo, Rod

\footnotetext{
${ }^{26}$ El GTAP es dirigido por Thomas Hertel.

${ }^{27}$ La información de TRAINS fue originalmente recopilada para utilizar en Berretoni y Cicowiez (2001).
} 
Tyers, and Thomas W. Hertel (1998) ${ }^{28}$. El procedimiento que siguen estos autores para estimar la participación de cada tipo de trabajo en la producción de cada sector consiste en estimar econometricamente la siguiente ecuación:

$$
M H P=F(G D P C, T E R)
$$

donde MHP es la participación del trabajo calificado en el pago total al factor trabajo, GDPC es el PBI per cápita y TER es el promedio de años de educación terciaria de toda la fuerza de trabajo de la economía.

Para la estimación utilizan información para 20 de las 30 regiones consideradas en la versión 3 de la base de datos del GTAP. Con el resultado de la estimación, se predice el valor de $M H P$ para las regiones no consideradas en la muestra. En particular, la información para Argentina es estimada por lo que se la corrigió utilizando información obtenida de la $\mathrm{EPH}^{29}$.

Finalmente, para el cálculo del cambio en las tasas arancelarias sectoriales se utilizó información sobre comercio exterior argentino obtenida de TRAINS.

\section{B.2. ELASTICIDADES}

Las elasticidades de sustitución $\varepsilon_{\mathrm{i}}, \chi_{\mathrm{ij}} \mathrm{y} \xi_{\mathrm{i}}(D D$ versus $M D, D I$ versus $M I$ y $N$ versus $K$, respectivamente) se obtienen del GTAP. A su vez, estas elasticidades se originan en el Proyecto SALTER. Este proyecto realizó una extensa revisión de la literatura y algunas estimaciones propias para dar valor a las elasticidades sustitución de un modelo de CGE. Aunque se realiza un análisis de sensibilidad, esta es la fuente principal de las elasticidades empleadas en este trabajo. En la Tabla B1 se muestra el valor que asumen las elasticidades mencionadas.

\footnotetext{
${ }^{28}$ En ese trabajo, el objetivo final de los autores es utilizar el procedimiento para construir la versión 4 de la base de datos del GTAP.

${ }^{29}$ Debido a que la EPH es una encuesta urbana, el procedimiento descripto es particularmente importante para determinar el empleo de los distintos tipos de trabajo de los sectores primarios.
} 
Tabla B1: Elasticidades de sustitución

\begin{tabular}{lccc}
\hline \multicolumn{1}{c}{ Sector } & \multicolumn{2}{c}{ VA } & \multicolumn{2}{c}{ Armington } \\
& $\xi_{\mathrm{i}}$ & $\varepsilon_{\mathrm{i}}$ & $\chi_{\mathrm{ij}}$ \\
\hline Cereales y Ganadería & 0.50 & 2.44 & 2.44 \\
Otros Productos Agrícolas & 0.50 & 2.60 & 2.60 \\
Industrias Alimenticias & 1.10 & 2.38 & 2.38 \\
Textiles e Indumentaria & 1.25 & 3.30 & 3.30 \\
Manufacturas de Recursos Naturales & 1.25 & 3.00 & 3.00 \\
Minería, Petróleo y Carbón & 0.50 & 2.62 & 2.62 \\
Transporte & 1.25 & 5.20 & 5.20 \\
Industria Química y otras Industrias & 1.25 & 2.50 & 2.50 \\
Maquinaria y Productos Metálicos & 1.25 & 2.80 & 2.80 \\
Servicios & 1.35 & 2.05 & 2.05 \\
\hline Fuente: GTAP. & & &
\end{tabular}

La primera columna de la Tabla B1 reporta las elasticidades de sustitución en el nivel superior del valor agregado, $\xi_{\mathrm{i}}$, para cada uno de los sectores productivos considerados. La elasticidad de sustitución entre factores primarios determina la facilidad de la economía para alterar su mix productivo como respuesta a cambios en el precio relativo de los bienes. Se observa que los sectores primarios presentan una elasticidad de sustitución menor. Esto está relacionado con la utilización de la tierra como factor primario en estos sectores lo que determina una respuesta limitada de la oferta de estos sectores ${ }^{30}$. El mayor valor para esta elasticidad de sustitución se observa en el sector Servicios.

La Tabla B1 también reporta las elasticidades de sustitución entre bienes domésticos e importados en el consumo final e intermedio, $\varepsilon_{\mathrm{i}} \mathrm{y} \chi_{\mathrm{ij}}$, respectivamente. Estas elasticidades son utilizadas en la estructura de demanda tipo Armington del Modelo General. Estas elasticidades determinan la facilidad con que los bienes domésticos pueden ser sustituidos por importaciones.

Siguiendo a Harrison et al. (1997) y Hillberry et al. (2001), en la especificación central del Modelo General, se emplea el doble de las elasticidades de sustitución Armington del GTAP. Estos autores, entre otros, concluyen que las elasticidades de sustitución Armington del GTAP son bajas, en especial para simulaciones que analizan extensos períodos de tiempo

\footnotetext{
${ }^{30}$ En este trabajo, el factor tierra está incluido en el factor capital.
} 


\section{APENDICE C: SIMULACIONES ADICIONALES}

En este apéndice se utiliza el Modelo General para analizar la apertura comercial argentina pero diferenciando por tipo de importación.

Utilizando los parámetros de la especificación central, se realizan tres simulaciones adicionales: En la primera, sólo se simula la baja de los aranceles a la importación de bienes de capital ${ }^{31}$. En la segunda, sólo se simula la baja de los aranceles a la importación de insumos intermedios $^{32}$. Por último, en la tercera, sólo se simula la baja de aranceles a la importación de bienes destinados al consumo final.

En la Tabla C1 aparecen los resultados. La primera columna reproduce el ejercicio 1 de la Sección VI y se incluye para facilitar la comparación de los resultados.

Tabla C1: Resultados

\begin{tabular}{lcccc}
\hline & EJ 1 & $\begin{array}{c}\text { Bienes de } \\
\text { Capital }\end{array}$ & $\begin{array}{c}\text { Consumo } \\
\text { Intermedio }\end{array}$ & $\begin{array}{c}\text { Consumo } \\
\text { Final }\end{array}$ \\
\hline $\begin{array}{l}\text { Porcentaje del cambio } \\
\text { en } w_{H} / w_{L} \text { debido a la } \\
\text { apertura comercial }\end{array}$ & 2.9 & 0.6 & 1.3 & 1.0 \\
$\begin{array}{l}\text { Porcentaje del cambio } \\
\text { en } w_{H} / w_{L} \text { debido a }\end{array}$ & 97.1 & 99.4 & 98.7 & 99.0 \\
otros factores & & & & \\
\hline
\end{tabular}

El resultado que se obtiene es que la mayor parte del aumento en el diferencial salarial se debe a la baja de aranceles a la importación de insumos intermedios. La baja de aranceles al consumo intermedio logra explicar 1,3\% del aumento total en el diferencial salarial. Se observa también que la baja de aranceles a la importación de bienes de capital explica 0,6\% del aumento total del diferencial salarial. Finalmente, la apertura comercial por el lado del consumo final logra explicar 1\% el aumento total del diferencial salarial.

\footnotetext{
${ }^{31}$ En este ejercicio, se simula una baja de aranceles al consumo intermedio de importaciones de Maquinaria y Productos Metálicos.

${ }^{32}$ Se simula como una disminución de los aranceles al consumo intermedio de importaciones de todos los bienes excepto Maquinaria y Productos Metálicos.
} 


\section{APENDICE D: CORRIDAS DEL MODELO}

Para determinar el cambio tecnológico de manera residual para que el modelo replique el incremento del diferencial salarial ocurrido entre 1992 y 1998, los modelos presentados en la Sección IV son resueltos cinco veces. La Tabla D1 muestra cuáles son las variables endógenas y cuáles las exógenas en cada una de las cinco corridas del modelo. Aunque la tabla se refiere al Modelo Simple, con muy pocas modificaciones puede ser adaptada al Modelo General.

Tabla D1: Descripción de cada corrida del modelo

\begin{tabular}{|c|c|c|c|c|}
\hline Corrida & Descripción & Objetivo & V. Endógenas & V. Exógenas \\
\hline 1 & $\begin{array}{l}\text { Réplica del caso } \\
\text { base }\end{array}$ & $\begin{array}{l}\text { Verificar la } \\
\text { correcta } \\
\text { codificación del } \\
\text { modelo }\end{array}$ & $\begin{array}{l}\mathrm{W}_{\mathrm{H}}, \mathrm{w}_{\mathrm{L}}, \mathrm{P}_{\mathrm{D}}, \\
\mathrm{L}_{\mathrm{D}}, \mathrm{H}_{\mathrm{D}}, \mathrm{L}_{\mathrm{E}}, \\
\mathrm{H}_{\mathrm{E}}, \mathrm{D}^{\mathrm{s}}, \mathrm{E}^{\mathrm{s}}, \mathrm{E}^{\mathrm{d}}, \\
\mathrm{D}^{\mathrm{d}}, \mathrm{M}\end{array}$ & $\begin{array}{l}P_{E}, P_{M}, L, H, \\
\tau, \pi\end{array}$ \\
\hline 2 & $\begin{array}{l}\text { Solución sólo con } \\
\text { cambios en } \tau\end{array}$ & $\begin{array}{l}\text { Calcular el efecto } \\
\text { sobre los salarios } \\
\text { del cambio en el } \\
\text { arancel }\left(\tau^{\prime}\right)\end{array}$ & Idem 1 & $\begin{array}{l}P_{E}, P_{M}, L, H, \\
\tau^{\prime}, \pi\end{array}$ \\
\hline 3 & $\begin{array}{l}\text { Solución con } \\
\text { cambios en } \tau \text { y se } \\
\text { determina el } \\
\text { cambio } \\
\text { tecnológico }\end{array}$ & $\begin{array}{l}\text { Calcular el valor } \\
\text { del cambio } \\
\text { tecnológico }(\pi *) \\
\text { consistente con el } \\
\text { cambio observado } \\
\text { en } \mathrm{w}_{\mathrm{H}} / \mathrm{w}_{\mathrm{L}}\end{array}$ & $\begin{array}{l}\text { Idem } 1+\pi y \\
\text { se obtiene } \pi^{*}\end{array}$ & $\tau^{\prime}, \mathrm{w}_{\mathrm{H}} / \mathrm{w}_{\mathrm{L}}$ \\
\hline 4 & $\begin{array}{l}\text { Solución sólo con } \\
\text { cambio } \\
\text { tecnológico }\end{array}$ & $\begin{array}{l}\text { Calcular el efecto } \\
\text { sobre los salarios } \\
\text { del cambio } \\
\text { tecnológico }\end{array}$ & Idem 1 & $\tau, \pi^{*}$ \\
\hline 5 & $\begin{array}{l}\text { Solución con } \\
\text { cambios en } \tau \text { y } \\
\text { cambio } \\
\text { tecnológico }\end{array}$ & $\begin{array}{l}\text { Comprobar que los } \\
\text { resultados son } \\
\text { correctos }\end{array}$ & Idem 1 & $\tau^{\prime}, \pi^{*}$ \\
\hline
\end{tabular}

Referencia: $\tau$ es el arancel en el equilibrio inicial y $\tau$ ' es el arancel en el contrafactual. 